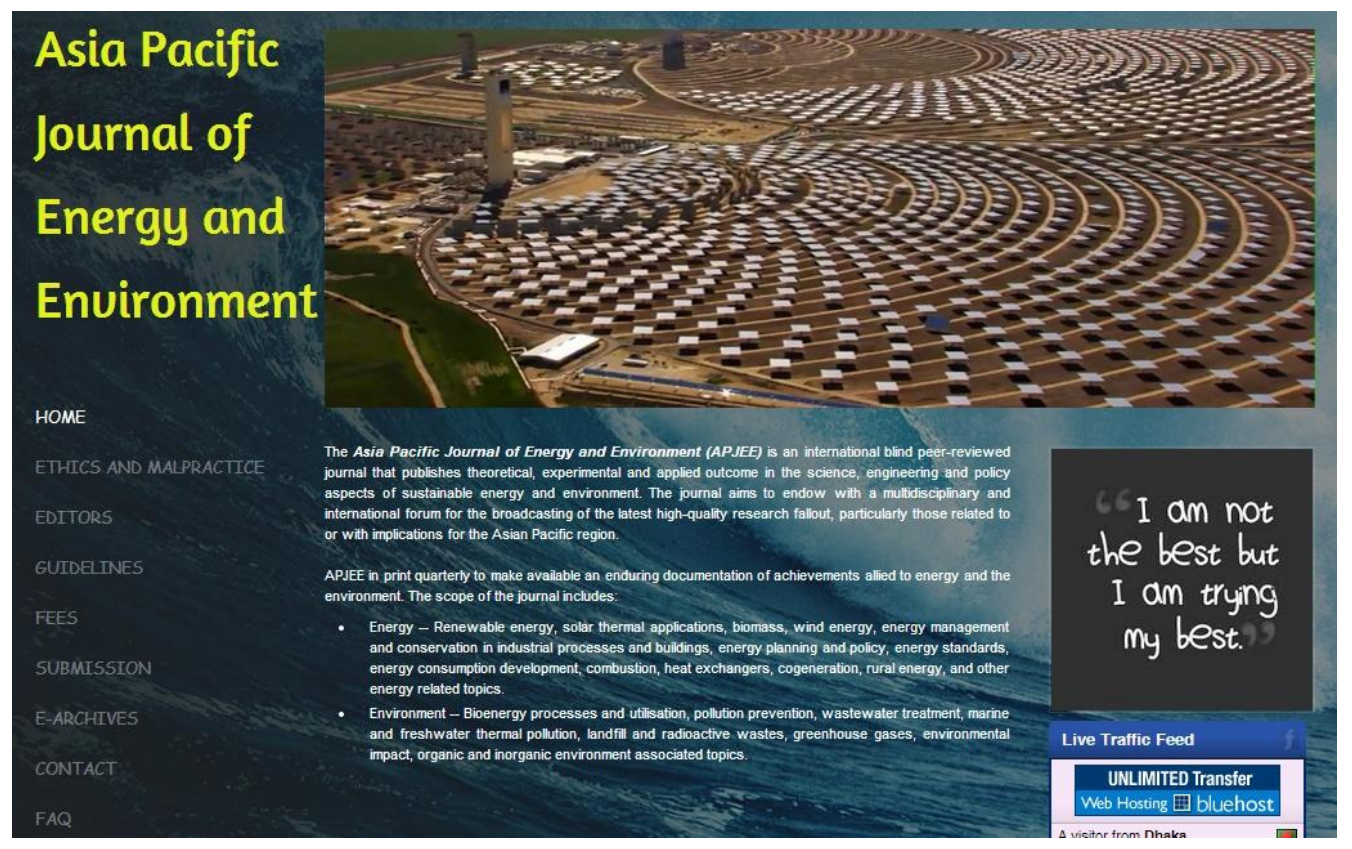




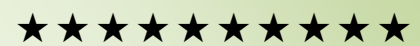

\section{ABO Journals}

- Online Submission

- Peer Reviewed

- Open Access

- Online Archives

- Paperless Review

- Prompt Feedback

- Global Circulation

- International Authorship

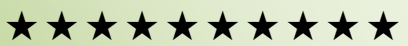




\title{
Petrography and Petrochemical Characteristics of Rare Metal Pegmatites around Oro, Southwestern Nigeria
}

\section{Oyebamiji Abiola 0.}

Mineral Exploration, University of Ibadan, Nigeria

\begin{abstract}
Petrographic and geochemical evaluation of pegmatite bodies occurring as discontinuous dykes have been studied in Oro area of southwestern Nigeria, with a view to determine the compositional characteristics that may be related to $\mathrm{Ta}-\mathrm{Nb}$ mineralization potentials. They are found intruding semi discordantly, the older assemblages of quartz mica schist and granite gneiss. A total of twenty-eight samples comprising whole rock pegmatites and eluvial soils were analyzed for major, trace and rare earth elements using (ICP-AES) geochemical method which show the pegmatite samples to be generally highly siliceous with $\mathrm{SiO} 2$ values ranging from $(71.49-97.77 \%)$ with an average of $86.43 \%$ in the whole rock samples, and it also ranges from (41.88-57.99\%) with an average of $48.43 \%$ in the eluvial soil samples, while $\mathrm{Al} 2 \mathrm{O} 3$ content is as high as $25.87 \%$ in the eluvial soil samples and $6.86 \%$ in the whole rock samples depicting the complexity of the pegmatite rock.

Variation plots of $K / R b$ versus $R b$, Ta versus $R b$, Ta versus $N b$, Ta versus $\mathrm{K} / \mathrm{Cs}$, Ta versus $\mathrm{Cs}+\mathrm{Rb}$ and $\mathrm{Ta}$ versus $\mathrm{Cs}$ discriminant plot indicate the potentials of rare metal mineralization.
\end{abstract}

Keywords: Pegmatite, Eluvial soils, Mineralization, Geochemical

\section{INTRODUCTION}

The study area lies within longitude $4050^{\prime} 00^{\prime \prime} \mathrm{E}-4052^{\prime} 00^{\prime \prime} \mathrm{E}$ and latitude $8^{0} 09^{\prime} 00^{\prime \prime} \mathrm{N}$ $8^{0} 16^{\prime} 00^{\prime \prime} \mathrm{N}$ (Ilorin sheet $223 \mathrm{NW}$ ) was studied as a result of recent increase in global demand for rare-metals pegmatites due to their ability to host many metallic, non-metallic or industrial minerals and gem mineralization, especially, those that are of immense economic importance. Oro area is located in the southwestern part of Nigeria. The area is underlain by the basement complex rocks of Nigeria, which comprises quartz mica schist and granite gneisses intruded by pegmatites veins. The Oro pegmatites occur as discordant low lying dykes intruding the host rocks which are already fairly weathered.

The Precambrian pegmatites of Nigeria are known to host variety rare metals, namely tantalum, niobium, tin, tungsten, columbite, lithium as well as gem stones (beryl, tourmaline, garnet), mica, feldspar and quartz which are presently in high demand globally. In recent times, there has been a resurgence of interest in the study of these pegmatites occurrences because of their associated economic rare metal and gem 
mineralization. These have led to concentration of study on discrimination of the pegmatites into rare metal mineralized and barren ones in order to elucidate modes and features of mineralization (Matheis, 1981; Matheis et.al. 1982; Kuster, 1990; Garba, 2003). Recent studies by Okunlola, (2005) also defined the metallogeny of the rare metal Ta-Nb pegmatites of Nigeria outlining seven (7) broad fields namely Kabba-Isanlu, Ijero-Aramoko, Keffi-Nasarawa, LemaNdeji, Oke Ogun, Ibadan- Oshogbo and Kushaka-Birnin Gwari. The Oro pegmatites occurrences which are members of the Kabba-Isanlu field occurrences have therefore been studied with the aim of elucidating their petrography and geochemical features and thus understanding their origin economic potentials in relation to other fields.

Researchers like Moller and Morteani (1987), Cerny (1989), Kuster (1990), Garba (2003) have contributed to a better understanding of southwestern and northern Nigeria pegmatite bodies distinguishing between the barren and rare metal bearing pegmatites and documenting that the pegmatites are not confined to the earlier proposed $400 \mathrm{~km}$ long NE-SW trending belt stretching from Wamba area (near Jos plateau) to Ilesha area. Elueze et. al. (2004) documented the industrial properties of the Olode-Falansa pegmatites in southwestern Nigeria. Okunlola and Ocan (2002) studied the Kabba mines environment, central Nigeria to look at the expected effect of the mining activities on the environment. Okunlola and Oyedokun (2009) studied the compositional trends and rare metal mineralization potential of Igbeti pegmatites southwestern Nigeria. Okunlola and King (2003) documented the process test work recovery of tantalite from Nasarawa area, central Nigeria. Other workers include; Akintola et. al. (2011) and Akintola et. al. (2012) described the compositional trends and rare metal mineralization potential of Precambrian pegmatites of Komu and Ago-Iwoye areas of southwestern Nigeria.

\section{Materials AND Methods}

The study involves systematic geological mapping on a scale of 1: 50,000 and thin section examinations of ten (10) whole rock pegmatites samples. A total of eighteen (18) eluvial soils samples were collected making the most widely available pegmatite occurrence in the study area. The sampling interval varied unavoidably as interest is on the exposures of the pegmatite rocks, therefore the sampling pattern was largely dictated by accessibility and geometry of the study area. The eluvial soil samples were collected by employing the interval sampling technique in which samples were collected at depth of $1 \mathrm{~m}, 2 \mathrm{~m}$ and $3 \mathrm{~m}$ respectively while channel sampling technique was used to collect composite samples along the downhole exposures of the pits up to a depth of $5 \mathrm{~m}$. The actual sampling points were determined with the use of Global Positioning System (GPS) and later plotted on the topographical map of the study area.

The whole rock pegmatite samples as well as the eluvial soil samples were analysed for major and trace elements at the Acme Analytical Laboratories Limited, Vancouver, British Columbia Canada using the Inductively Coupled Plasma Atomic Emission Spectrometry (ICP-AES) instrumentation method. The major elements are given in weight percent $(\%)$, while the trace elements are given in parts per million ( $\mathrm{ppm})$. The procedure employed involves digestion of representative sample of whole rock pegmatite and eluvial soils using nitric acid. About $0.5 \mathrm{~g}$ of each sample was measured into the dry digested tube. 3-4 drops of distilled water was added to the wet sample. $5 \mathrm{ml}$ of $\mathrm{HCl}$ was added to wet the sample and the solution was stirred. $5 \mathrm{ml}$ of nitric/perchloric acid was added in the ratio 3:2 and stirred. The digesting tube was left overnight heating. The samples were leached out with $6 \mathrm{ml} \mathrm{HCl}$ in a tube and made up to $20 \mathrm{ml}$ mark with distil water.

To avoid caking, the content was shaken vigorously and the resulting solution is referred to as stock solution. The stock solution was used directly to determine the elements. It is 
becoming increasingly accepted as a tool for trace element and isotopic analysis as a result of the very low detection limits, good accuracy and precision. This technique consist of sample introduction system, referred to as Nebulizer, the ICP torch, the high frequency generator, the transfer optics, the spectrometer, the interface and computer.

A digested solution of the sample to be analyzed is introduced into the ICP torch as an aqueous aerosol, the light emitted by the atoms or ions in the ICP is converted to an electrical signal by a photomultiplier in the spectrometer. The intensity of this electrical signal is compared to a previously measured intensity of a known concentration of the element and the concentration value is then computed.

Fig.1: Sample location map of the study area showing drainage pattern and sampling points.

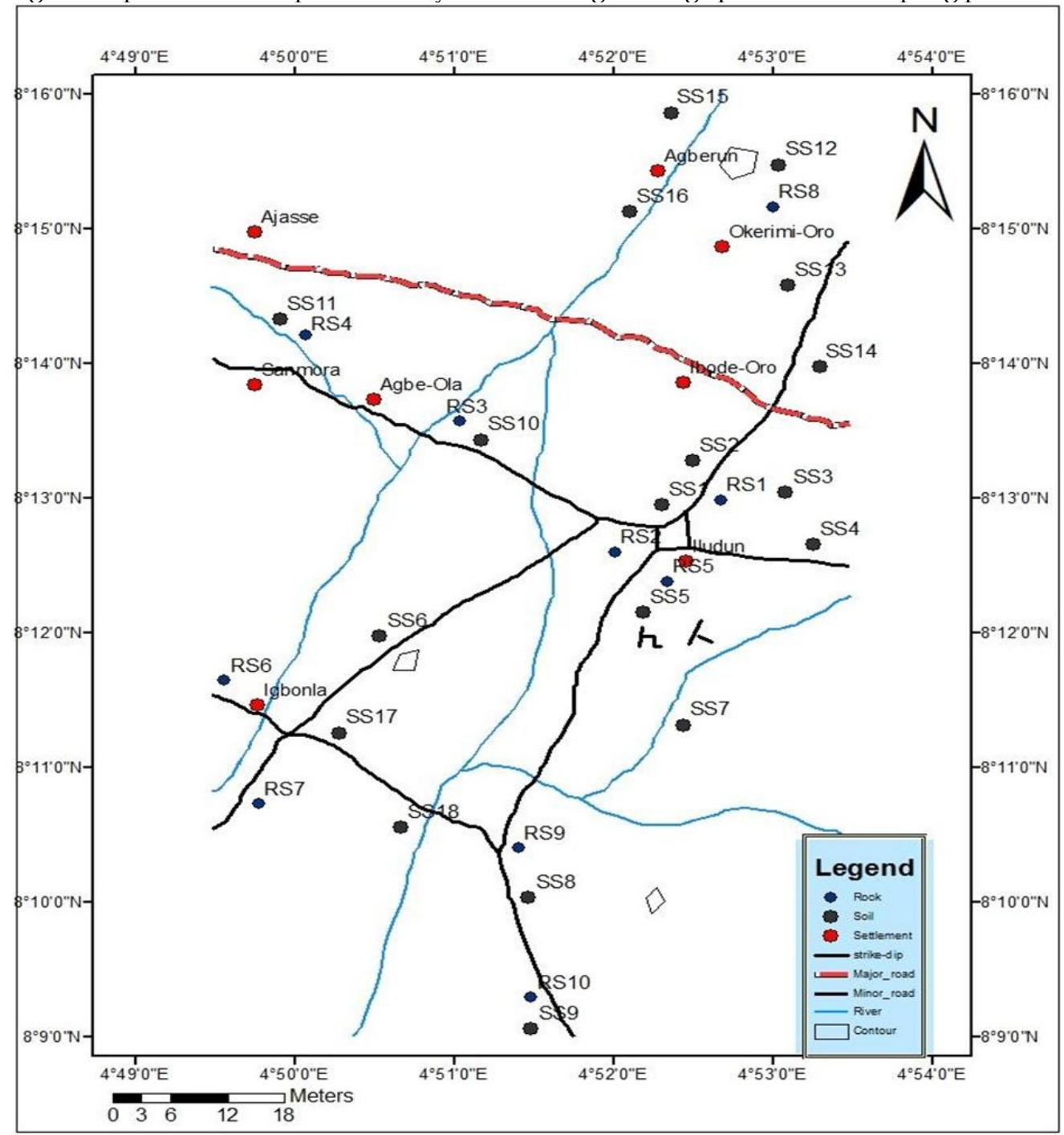




\section{RESULTS AND DISCUSSION}

The study area is underlain by quartz mica schists and granite gneisses, all intruded by pegmatites. The rocks are very sparse in the area and most of those mapped are already fairly weathered. The schists often occur as layered relicts, lensoidal or pools of pods. Most of the original sedimentary features have been obliterated by poly phase metamorphism. They are mainly quartz mica schists, mostly light brownish to dark grey in colour. They are exposed sporadically within the pervasive pegmatite zone in virtually the whole area as horizontally -dipping oral bodies inter layered with the rare metal bearing pegmatite. The granite gneiss occurs mostly in the southwestern corner of the area. It is weakly foliated but poorly developed mineral lineation and banding on millimeter scale. Quartz, feldspar, biotite are the main minerals with quartz being the most abundant. The pegmatites of this area are obviously members of the larger NE-SW trending belt extending from Abeokuta to Wamba-Jemaa area in central Nigeria as designated by Jacobson and Webbs (1946). The main mineral assemblages are albite, microcline, quartz, muscovite, sericite, black tourmaline (shorl), columbite-tantalite. Albite is the most abundant of these minerals and forms coarse crystalline graphic intergrowth with quartz (Fig. 2). They are sometimes perthitic with microcline as patchy and feathery perthites. These may indicate local metasomatic replacement. Most of the microcline display characteristic crosshatch twinning. It occurs as irregular grains varying from colourless to milky white with occasional yellow stains. It is medium to coarse grained.

Fig. 2: Photomicrograph of Pegmatite in transmitted light showing Microcline (Mi), Quartz (Q), Biotite (B) and Albite (A)

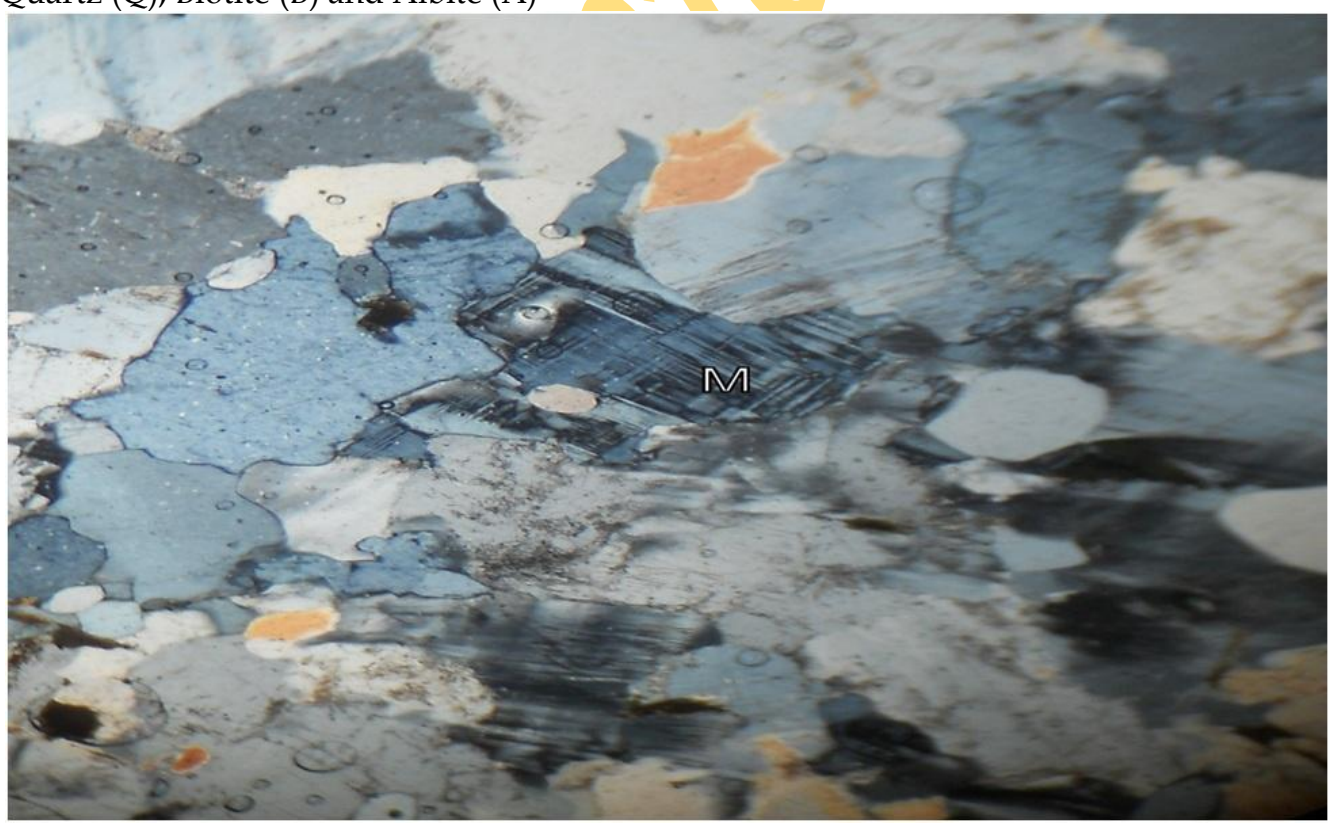

Fig 3: Geological map of the study area 


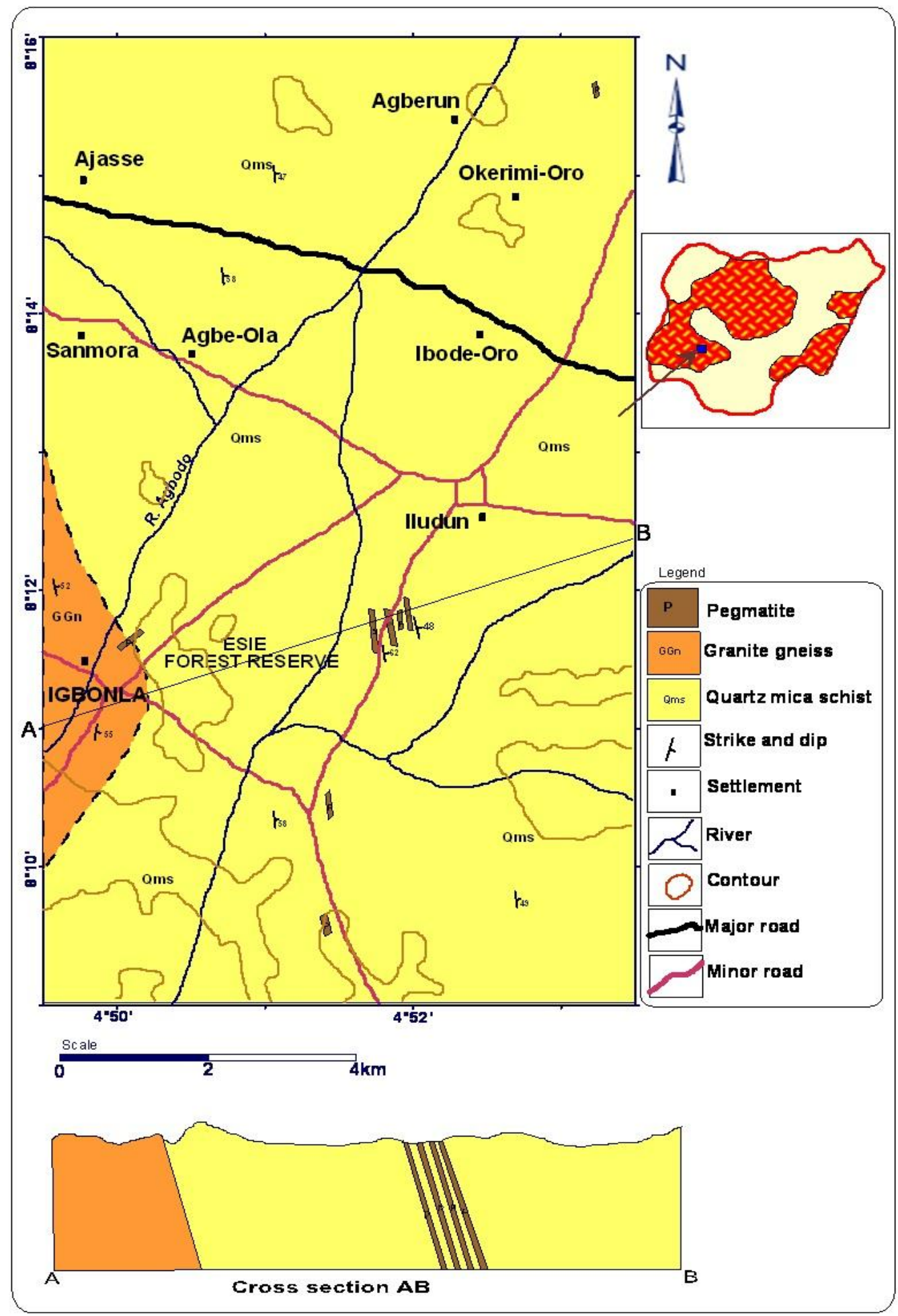


The analytical results of the major oxide composition as presented in (Table 2), it shows that the samples of the Oro pegmatite are highly siliceous with $\mathrm{SiO}_{2}$ content ranging from 71.49-97.77\% with an average of $86.43 \%$ in the Oro whole rock pegmatite samples, the $\mathrm{Al}_{2} \mathrm{O}_{3}$ ranges from $0.73-15.20 \%$ with an average of $6.86 \%$, also the $\mathrm{Fe}_{2} \mathrm{O}_{3}$ ranges from $0.45-6.09 \%$ with a mean value of $2.05 \%$. The range and mean contents of other major oxides in the whole rock pegmatites of Oro are as follows; $\mathrm{MnO}(<0.01-0.04 \%, 0.03 \%), \mathrm{MgO}(0.01-1.32 \%, 0.23 \%), \mathrm{CaO}(<0.01-0.65 \%$, $0.22 \%), \mathrm{Na}_{2} \mathrm{O}(0.02-6.82 \%, 2.09 \%), \mathrm{K}_{2} \mathrm{O}(0.09-0.99 \%, 0.45 \%), \mathrm{TiO}_{2}(0.02-0.87 \%, 0.30 \%), \mathrm{P}_{2} \mathrm{O}_{5}(0.01-$ $0.18 \%, 0.08 \%)$ and $\mathrm{Cr}_{2} \mathrm{O}_{3}(<0.002-0.013 \%, 0.006 \%)$. Also from the analytical results of the major oxide composition as presented in (Table 3), it shows that the eluvial soil samples around the Oro pegmatite are moderately siliceous with $\mathrm{SiO}_{2}$ content ranging from $41.88-57.99 \%$ with a mean value of $48.43 \%$, the $\mathrm{Al}_{2} \mathrm{O}_{3}$ ranges from $17.79-34.94 \%$ with an average of $25.87 \%$ in the eluvial soil around the pegmatites. This slight to sharp contrast in the values of some of the whole rock and eluvial soils for the alumina content of this study area in addition with other rare metal characteristics, confirms the complexity of the pegmatite type.

These values are comparable to those observed for mineralized pegmatites of Nigeria, (Garba, 2003; Okunlola, 2005).

Table 2: Major oxide composition of Oro whole rock pegmatites (wt \%)

\begin{tabular}{|c|c|c|c|c|c|c|c|c|c|c|}
\hline Oxides (\%) & RS1 & RS2 & RS3 & RS4 & RS5 & RS6 & RS7 & RS8 & RS9 & RS10 \\
\hline $\mathrm{SiO} 2$ & 97.77 & 96.59 & 92.40 & 92.92 & 88.99 & 75.48 & 74.54 & 77.60 & 71.49 & 96.49 \\
\hline $\mathrm{A} 2 \mathrm{O} 3$ & 0.73 & 1.18 & 2.15 & 2.01 & 5.75 & 13.58 & 15.20 & 13.30 & 13.44 & 1.26 \\
\hline $\mathrm{Fe} 2 \mathrm{O} 3$ & 0.45 & 0.95 & 2.90 & 2.65 & 1.99 & 2.86 & 0.79 & 0.78 & 6.09 & 1.04 \\
\hline $\mathrm{CaO}$ & $<0.01$ & 0.01 & 0.02 & 0.02 & 0.02 & 0.51 & 0.65 & 0.57 & 0.4 & 0.02 \\
\hline $\mathrm{MgO}$ & 0.01 & 0.02 & 0.05 & 0.05 & 0.05 & 0.59 & 0.07 & 0.06 & 1.32 & 0.03 \\
\hline $\mathrm{Na} 2 \mathrm{O}$ & 0.02 & 0.02 & 0.02 & 0.02 & 0.15 & 4.83 & 6.82 & 5.86 & 3.17 & 0.02 \\
\hline $\mathrm{K} 2 \mathrm{O}$ & 0.09 & 0.09 & 0.23 & 0.22 & 0.89 & 0.58 & 0.99 & 0.87 & 0.39 & 0.09 \\
\hline $\mathrm{TiO} 2$ & 0.06 & 0.06 & 0.67 & 0.56 & 0.07 & 0.37 & 0.38 & 0.02 & 0.87 & 0.07 \\
\hline $\mathrm{P} 2 \mathrm{O} 5$ & 0.04 & 0.02 & 0.01 & 0.02 & 0.03 & 0.14 & 0.18 & 0.17 & 0.17 & 0.03 \\
\hline $\mathrm{MnO}$ & 0.02 & 0.03 & 0.04 & 0.04 & $<0.01$ & 0.03 & 0.03 & 0.03 & 0.03 & 0.04 \\
\hline $\mathrm{Cr} 2 \mathrm{O} 3$ & 0.003 & 0.004 & 0.013 & 0.012 & 0.003 & 0.006 & $<0.002$ & 0.003 & 0.012 & 0.003 \\
\hline $\mathrm{LOI}$ & 0.6 & 1.0 & 1.5 & 1.5 & 2.1 & 1.0 & 0.7 & 0.8 & 2.5 & 0.9 \\
\hline Total & 99.79 & 99.97 & 100.00 & 100.04 & 100.04 & 99.08 & 100.35 & 100.06 & 99.88 & 99.99 \\
\hline
\end{tabular}

Table 3: Major oxide composition of eluvial soils in some pits around the Oro pegmatites (wt \%)

\begin{tabular}{|c|c|c|c|c|c|c|c|c|c|c|}
\hline Oxides (\%) & SS1 & SS2 & SS3 & SS4 & SS5 & SS6 & SS7 & SS8 & SS9 & SS10 \\
\hline SiO2 & 47.30 & 49.04 & 48.55 & 57.99 & 47.04 & 41.88 & 50.27 & 47.56 & 46.13 & 43.47 \\
\hline Al2O3 & 26.38 & 26.79 & 23.65 & 23.61 & 34.94 & 33.31 & 26.38 & 23.07 & 25.35 & 34.15 \\
\hline Fe2O3 & 8.60 & 9.20 & 10.20 & 4.82 & 2.35 & 4.08 & 7.58 & 10.64 & 8.60 & 4.17 \\
\hline CaO & 0.06 & 0.05 & 0.15 & 0.02 & 0.05 & 0.05 & 0.07 & 0.17 & 0.06 & 0.05 \\
\hline MgO & 0.19 & 0.23 & 2.57 & 0.07 & 0.08 & 0.15 & 0.18 & 3.08 & 0.36 & 0.15 \\
\hline Na2O & 0.05 & 0.02 & 0.07 & $<0.01$ & $<0.01$ & $<0.01$ & 0.04 & 0.06 & 0.04 & $<0.01$ \\
\hline K2O & 1.07 & 0.67 & 1.83 & 0.42 & 0.56 & 0.26 & 0.89 & 2.06 & 0.99 & 0.26 \\
\hline TiO2 & 1.20 & 0.85 & 0.77 & 0.41 & 0.11 & 0.13 & 0.92 & 0.74 & 1.10 & 0.12 \\
\hline P2O5 & 0.07 & 0.05 & 0.07 & 0.06 & 0.12 & 0.05 & 0.05 & 0.07 & 0.06 & 0.04 \\
\hline MnO & 0.12 & 0.03 & 0.10 & 0.02 & 0.02 & $<0.01$ & 0.08 & 0.12 & 0.11 & $<0.01$ \\
\hline Cr2O3 & 0.013 & 0.016 & 0.014 & 0.007 & 0.004 & 0.006 & 0.012 & 0.014 & 0.012 & 0.006 \\
\hline LOI & 14.7 & 12.9 & 11.7 & 12.6 & 14.2 & 20.0 & 13.4 & 12.0 & 17.0 & 17.5 \\
\hline Total & 99.75 & 99.85 & 99.60 & 100.01 & 99.47 & 99.92 & 99.87 & 99.58 & 99.81 & 99.92 \\
\hline
\end{tabular}

\begin{tabular}{|c|c|c|c|c|c|c|c|c|}
\hline Oxides (\%) & SS11 & SS12 & SS13 & SS14 & SS15 & SS16 & SS17 & SS18 \\
\hline SiO2 & 48.99 & 42.50 & 48.91 & 45.15 & 51.04 & 54.87 & 54.94 & 46.07 \\
\hline Al2O3 & 22.98 & 27.20 & 26.99 & 27.72 & 26.78 & 17.94 & 17.79 & 20.62 \\
\hline Fe2O3 & 9.98 & 9.69 & 9.11 & 9.68 & 6.14 & 2.69 & 2.71 & 12.68 \\
\hline $\mathrm{CaO}$ & 0.19 & 0.05 & 0.05 & 0.08 & 0.06 & 5.43 & 5.42 & 0.21 \\
\hline $\mathrm{MgO}$ & 2.60 & 0.13 & 0.18 & 1.02 & 0.12 & 0.73 & 0.73 & 5.09 \\
\hline
\end{tabular}


Asia Pacific Journal of Energy and Environment, Volume 1, No 1 (2014)

\begin{tabular}{|c|c|c|c|c|c|c|c|c|}
\hline Na2O & 0.08 & 0.02 & 0.02 & 0.04 & 0.05 & 0.04 & 0.04 & 0.08 \\
\hline K2O & 1.91 & 0.75 & 0.65 & 1.15 & 0.98 & 3.03 & 3.02 & 2.91 \\
\hline TiO2 & 0.78 & 0.89 & 0.86 & 0.85 & 0.65 & 0.90 & 0.90 & 0.72 \\
\hline P2O5 & 0.07 & 0.06 & 0.06 & 0.07 & 0.07 & 0.19 & 0.18 & 0.07 \\
\hline MnO & 0.11 & 0.08 & 0.03 & 0.09 & 0.05 & 0.02 & 0.02 & 0.14 \\
\hline Cr2O3 & 0.014 & 0.012 & 0.016 & 0.012 & 0.009 & 0.006 & 0.007 & 0.016 \\
\hline LOI & 12.0 & 18.5 & 13.0 & 15.9 & 13.9 & 10.6 & 10.7 & 10.9 \\
\hline Total & 99.70 & 99.88 & 99.88 & 101.76 & 99.85 & 96.45 & 96.46 & 99.51 \\
\hline
\end{tabular}

Table 4: Range and average values of major oxides in the whole rock and eluvial soils in some pits around the Oro pegmatites in mass fraction (wt \%)

\begin{tabular}{|c|c|c|c|c|}
\hline Major Elements & \multicolumn{2}{|c|}{ Whole rock pegmatite } & \multicolumn{2}{|l|}{ Eluvial soils } \\
\hline & $\mathrm{n}=10$ & & $\mathrm{n}=18$ & \\
\hline & Range & Average & Range & Average \\
\hline $\mathrm{SiO}_{2}$ & $71.49-97.77$ & 86.43 & $41.88-57.99$ & 48.43 \\
\hline $\mathrm{Al}_{2} \mathrm{O}_{3}$ & $0.73-15.20$ & 6.86 & $17.79-34.94$ & 25.87 \\
\hline $\mathrm{Fe}_{2} \mathrm{O}_{3}$ & $0.45-6.09$ & 2.05 & $2.35-12.68$ & 7.38 \\
\hline $\mathrm{CaO}$ & $<0.01-0.65$ & 0.22 & $0.02-5.43$ & 0.68 \\
\hline $\mathrm{MgO}$ & $0.01-1.32$ & 0.23 & $0.07-5.09$ & 0.98 \\
\hline $\mathrm{Na}_{2} \mathrm{O}$ & $0.02-6.82$ & 2.09 & $<0.01-0.08$ & 0.04 \\
\hline $\mathrm{K}_{2} \mathrm{O}$ & $0.09-0.99$ & 0.45 & $0.26-3.03$ & 1.30 \\
\hline $\mathrm{TiO}_{2}$ & $0.02-0.87$ & 0.30 & $0.11-1.20$ & 0.72 \\
\hline $\mathrm{P}_{2} \mathrm{O}_{5}$ & $0.01-0.18$ & 0.08 & $0.04-0.19$ & 0.08 \\
\hline $\mathrm{MnO}$ & $<0.01-0.04$ & 0.03 & $<0.01-0.14$ & 0.06 \\
\hline $\mathrm{Cr}_{2} \mathrm{O}_{3}$ & $<0.002-0.013$ & 0.006 & $0.004-0.016$ & 0.011 \\
\hline
\end{tabular}

Table 5: Trace and rare earth elements composition of Oro whole rock pegmatites (ppm)

\begin{tabular}{|c|c|c|c|c|c|c|c|c|c|c|}
\hline Elements & RS1 & RS2 & RS3 & RS4 & RS5 & RS6 & RS7 & RS8 & RS9 & RS10 \\
\hline $\mathrm{Ag}$ & $<0.1$ & $<0.1$ & $<0.1$ & $<0.1$ & $<0.1$ & $<0.1$ & $<0.1$ & $<0.1$ & $<0.1$ & $<0.1$ \\
\hline As & $<0.5$ & 0.5 & $<0.5$ & $<0.5$ & 0.7 & $<0.5$ & $<0.5$ & $<0.5$ & $<0.5$ & 1.0 \\
\hline $\mathrm{Au}$ & 4.8 & 2.9 & 1.7 & 3.8 & 2.2 & 1.2 & 0.9 & 1.5 & $<0.5$ & $<0.5$ \\
\hline $\mathbf{B a}$ & 38 & 72 & 64 & 64 & 26 & 51 & 19 & 23 & 143 & 90 \\
\hline Be & $<1$ & $<1$ & $<1$ & $<1$ & 25 & 4 & 9 & 3 & 6 & $<1$ \\
\hline$\overline{B i}$ & $<0.1$ & $<0.1$ & $<0.1$ & $<0.1$ & 81 & 1.2 & 2.1 & 2.0 & 0.7 & $<0.1$ \\
\hline $\mathrm{Cd}$ & $<0.1$ & $<0.1$ & $<0.1$ & $<0.1$ & $<0.1$ & $<0.1$ & $<0.1$ & $<0.1$ & $<0.1$ & $<0.1$ \\
\hline $\mathrm{Ce}$ & 111.4 & 152.7 & 61.8 & 75.6 & 19.0 & 62.4 & 5.0 & 18.1 & 80.9 & 204.0 \\
\hline Co & 1.8 & 5.1 & 4.7 & 4.3 & 1.7 & 5.9 & 1.0 & 1.3 & 14.1 & 5.6 \\
\hline Cs & 0.1 & 0.3 & 1.2 & 1.1 & 7.1 & 4.4 & 6.0 & 5.5 & 2.8 & 0.5 \\
\hline $\mathrm{Cu}$ & 6.9 & 12.0 & 21.1 & 20.9 & 18.0 & 9.9 & 6.8 & 5.3 & 19.6 & 10.4 \\
\hline Dy & 0.17 & 0.45 & 3.17 & 2.63 & 0.67 & 1.80 & 2.44 & 1.01 & 3.86 & 0.50 \\
\hline Er & 0.05 & 0.14 & 2.09 & 1.52 & 0.22 & 0.67 & 1.01 & 0.42 & 1.84 & 0.10 \\
\hline Eu & 0.10 & 0.26 & 0.36 & 0.39 & 0.28 & 0.61 & 0.03 & 0.03 & 1.24 & 0.28 \\
\hline $\mathrm{Ga}$ & 0.6 & 1.8 & 3.7 & 3.5 & 16.3 & 19.4 & 21.4 & 18.5 & 20.8 & 1.6 \\
\hline Gd & 0.66 & 1.04 & 2.88 & 3.23 & 0.69 & 2.37 & 1.23 & 0.61 & 4.54 & 1.00 \\
\hline Hf & 0.3 & 0.3 & 10.2 & 8.1 & 1.1 & 2.4 & 1.8 & 0.7 & 12.0 & 0.4 \\
\hline $\mathrm{Hg}$ & $<0.01$ & $<0.01$ & $<0.01$ & $<0.01$ & $<0.01$ & $<0.01$ & $<0.01$ & $<0.01$ & $<0.01$ & $<0.01$ \\
\hline Ho & 0.03 & 0.07 & 0.67 & 0.53 & 0.10 & 0.27 & 0.39 & 0.13 & 0.65 & 0.06 \\
\hline La & 9.7 & 26.7 & 23.7 & 31.5 & 23.4 & 34.7 & 2.6 & 3.1 & 28.9 & 29.3 \\
\hline $\mathbf{L i}$ & $<20$ & $<20$ & $<20$ & $<20$ & $<20$ & 21 & $<20$ & $<20$ & 43 & $<20$ \\
\hline Lu & $<0.01$ & 0.02 & 0.42 & 0.28 & 0.06 & 0.10 & 0.16 & 0.05 & 0.28 & 0.03 \\
\hline Mo & 5.6 & 7.5 & 0.3 & 0.3 & 0.3 & $<0.1$ & 0.2 & 0.9 & $<0.1$ & 9.9 \\
\hline $\mathrm{Nb}$ & 1.15 & 1.82 & 16.66 & 15.18 & 99.65 & 35.80 & 44.54 & 35.76 & 40.42 & 1.98 \\
\hline Nd & 8.2 & 12.0 & 20.7 & 29.0 & 10.1 & 24.9 & 1.7 & 1.4 & 31.7 & 12.6 \\
\hline $\mathrm{Ni}$ & 1.6 & 2.8 & 6.1 & 6.8 & 2.2 & 5.6 & 0.3 & 0.4 & 11.4 & 3.1 \\
\hline $\mathrm{Pb}$ & 10.9 & 8.5 & 3.2 & 3.4 & 2.7 & 4.7 & 5.4 & 2.8 & 1.2 & 9.4 \\
\hline $\operatorname{Pr}$ & 1.65 & 3.95 & 5.57 & 7.45 & 3.25 & 6.83 & 0.48 & 0.54 & 7.4 & 4.32 \\
\hline $\mathbf{R b}$ & 4.1 & 5.4 & 14.8 & 13.5 & 383.6 & 85.9 & 102.4 & 87.6 & 36.2 & 5.7 \\
\hline $\mathrm{Sb}$ & 0.2 & $<0.1$ & $<0.1$ & $<0.1$ & $<0.1$ & $<0.1$ & 0.1 & $<0.1$ & $<0.1$ & $<0.1$ \\
\hline Sc & $<1$ & 1 & 7 & 6 & 2 & 4 & 1 & 1 & 10 & 1 \\
\hline Se & $<0.5$ & $<0.5$ & $<0.5$ & $<0.5$ & $<0.5$ & $<0.5$ & $<0.5$ & $<0.5$ & $<0.5$ & $<0.5$ \\
\hline
\end{tabular}


Asia Pacific Journal of Energy and Environment, Volume 1, No 1 (2014)

\begin{tabular}{|c|c|c|c|c|c|c|c|c|c|c|}
\hline Sm & 0.64 & 1.44 & 3.67 & 4.75 & 1.18 & 3.66 & 0.75 & 0.54 & 5.90 & 1.66 \\
\hline $\mathbf{S n}$ & 3.19 & $<1.06$ & 1.06 & 2.13 & 14.89 & 8.51 & 10.63 & 9.57 & 8.51 & $<1.06$ \\
\hline $\mathbf{S r}$ & 8.9 & 23.6 & 4.1 & 3.8 & 15.6 & 60.0 & 5.9 & 6.2 & 156.3 & 25.9 \\
\hline $\mathbf{T a}$ & 0.21 & 0.21 & 1.26 & 1.26 & 200.21 & 10.27 & 5.24 & 4.82 & 10.90 & 0.42 \\
\hline $\mathbf{T b}$ & 0.05 & 0.01 & 0.51 & 0.49 & 0.13 & 0.35 & 0.42 & 0.18 & 0.70 & 0.10 \\
\hline $\mathbf{T h}$ & 1.3 & 1.1 & 8.1 & 11.3 & 1.9 & 10.8 & 0.8 & 0.6 & 6.2 & 1.3 \\
\hline $\mathbf{T i}$ & 0.1 & 0.1 & $<0.1$ & 0.2 & $<0.1$ & 0.2 & 0.1 & 0.2 & $<0.1$ & 0.1 \\
\hline $\mathbf{T m}$ & $<0.01$ & 0.02 & 0.37 & 0.28 & 0.05 & 0.11 & 0.19 & 0.06 & 0.25 & 0.02 \\
\hline $\mathbf{U}$ & 0.2 & 0.3 & 0.9 & 0.8 & 4.4 & 3.3 & 5.3 & 3.7 & 2.1 & 0.3 \\
\hline $\mathbf{V}$ & $<8$ & 22 & 61 & 57 & 23 & 50 & $<8$ & $<8$ & 108 & 23 \\
\hline $\mathbf{W}$ & $<0.5$ & $<0.5$ & $<0.5$ & $<0.5$ & 0.9 & 0.8 & 0.5 & 0.6 & 0.9 & $<0.5$ \\
\hline $\mathbf{Y}$ & 0.7 & 1.4 & 18.9 & 12.1 & 2.6 & 7.0 & 12.7 & 4.7 & 15.5 & 1.5 \\
\hline $\mathbf{Y b}$ & 0.09 & 0.12 & 2.33 & 1.4 & 0.23 & 0.7 & 1.02 & 0.45 & 1.77 & 0.10 \\
\hline $\mathbf{Z n}$ & 5.0 & 7.0 & 7.0 & 6.0 & 23.0 & 40.0 & 47.0 & 43.0 & 39.0 & 6.0 \\
\hline $\mathbf{Z r}$ & 4.7 & 10.0 & 391.5 & 295.6 & 22.8 & 97.4 & 32.5 & 15.3 & 459.1 & 18.8 \\
\hline
\end{tabular}

Table 6: Trace and rare earth elements composition of eluvial soils in some pits around the Oro pegmatites (ppm)

\begin{tabular}{|c|c|c|c|c|c|c|c|c|c|c|}
\hline Elements & SS1 & SS2 & SS3 & SS4 & SS5 & SS6 & SS7 & SS8 & SS9 & SS10 \\
\hline $\mathrm{Ag}$ & $<0.1$ & $<0.1$ & $<0.1$ & $<0.1$ & $<0.1$ & $<0.1$ & $<0.1$ & $<0.1$ & $<0.1$ & $<0.1$ \\
\hline As & 1.2 & 2.3 & 1.0 & 1.2 & $<0.5$ & 0.9 & 1.4 & 0.9 & 1.3 & 0.8 \\
\hline $\mathbf{A u}$ & 1.2 & 1.8 & 2.2 & 1.5 & 0.7 & 1.0 & $<0.5$ & $<0.5$ & 1.6 & 1.3 \\
\hline $\mathbf{B a}$ & 232.0 & 166.0 & 656.0 & 101.0 & 92.0 & 63.0 & 182.0 & 762.0 & 258.0 & 63.0 \\
\hline Be & 112.0 & 24.0 & 39.0 & 38.0 & 381.0 & 29.0 & 59.0 & 29.0 & 84.0 & 23.0 \\
\hline $\mathbf{B i}$ & 2.9 & 28.2 & 11.0 & 10.7 & 1.0 & 1.0 & 2.5 & 7.4 & 3.2 & 1.1 \\
\hline $\mathrm{Cd}$ & $<0.1$ & $<0.1$ & $<0.1$ & $<0.1$ & $<0.1$ & $<0.1$ & $<0.1$ & $<0.1$ & $<0.1$ & $<0.1$ \\
\hline $\mathrm{Ce}$ & 151.9 & 51.8 & 125.3 & 46.8 & 55.6 & 22.4 & 101.5 & 165.7 & 150.4 & 22.0 \\
\hline Co & 12.3 & 8.4 & 21.5 & 4.3 & 1.4 & 2.4 & 12.3 & 24.2 & 12.0 & 2.1 \\
\hline Cs & 22.6 & 14.8 & 71.4 & 7.7 & 14.3 & 5.2 & 20.4 & 78.1 & 24.1 & 5.0 \\
\hline $\mathrm{Cu}$ & 32.2 & 20.0 & 98.5 & 13.8 & 8.8 & 13.4 & 30.8 & 107.1 & 34.7 & 12.4 \\
\hline Dy & 5.28 & 2.49 & 9.76 & 2.35 & 3.90 & 1.69 & 3.59 & 12.22 & 5.06 & 1.80 \\
\hline Er & 3.09 & 1.33 & 4.05 & 0.97 & 1.24 & 0.83 & 2.00 & 5.35 & 2.83 & 0.81 \\
\hline Eu & 1.63 & 0.98 & 5.56 & 0.83 & 1.33 & 0.66 & 1.17 & 6.80 & 1.81 & 0.65 \\
\hline $\mathrm{Ga}$ & 39.3 & 35.2 & 34.0 & 29.2 & 43.8 & 39.7 & 35.4 & 29.9 & 33.5 & 39.9 \\
\hline Gd & 5.98 & 3.60 & 18.99 & 2.92 & 5.24 & 2.59 & 4.69 & 21.76 & 7.01 & 2.38 \\
\hline Hf & 16.9 & 7.4 & 6.3 & 4.1 & 23.8 & 2.9 & 9.1 & 6.2 & 11.5 & 2.6 \\
\hline $\mathrm{Hg}$ & 0.01 & 0.02 & $<0.01$ & $<0.01$ & $<0.01$ & $<0.01$ & 0.02 & $<0.01$ & 0.02 & $<0.01$ \\
\hline Ho & 1.06 & 0.56 & 1.61 & 0.41 & 0.49 & 0.26 & 0.71 & 2.00 & 0.96 & 0.26 \\
\hline La & 68.8 & 49.8 & 265.0 & 34.6 & 50.4 & 25.2 & 50.3 & 330.3 & 79.7 & 25.0 \\
\hline $\mathrm{Li}$ & 51.0 & 46.0 & 88.0 & $<20.0$ & $<20.0$ & $<20.0$ & 51.0 & 81.0 & 53.0 & 26.0 \\
\hline Lu & 0.51 & 0.21 & 0.49 & 0.15 & 0.27 & 0.10 & 0.31 & 0.60 & 0.44 & 0.10 \\
\hline Mo & 1.0 & 1.4 & 5.7 & 0.5 & 0.1 & 0.3 & 0.7 & 7.4 & 1.3 & 0.4 \\
\hline $\mathrm{Nb}$ & 92.39 & 138.42 & 127.86 & 40.1 & 972.05 & 26.89 & 70.61 & 58.24 & 70.28 & 43.06 \\
\hline $\mathrm{Nd}$ & 51.5 & 35.8 & 231.8 & 26.6 & 49.3 & 20.2 & 39.0 & 295.6 & 64.8 & 19.7 \\
\hline $\mathrm{Ni}$ & 12.3 & 8.3 & 55.7 & 3.6 & 1.6 & 2.7 & 13.1 & 65.3 & 15.3 & 2.9 \\
\hline $\mathbf{P b}$ & 26.0 & 11.6 & 11.4 & 6.4 & 3.3 & 6.3 & 12.2 & 10.0 & 23.1 & 5.8 \\
\hline Pr & 14.78 & 9.72 & 66.81 & 7.64 & 13.67 & 5.69 & 10.32 & 81.63 & 18.15 & 5.74 \\
\hline $\mathbf{R b}$ & 261.7 & 172.1 & 364.2 & 88.7 & 136.2 & 58.3 & 233.1 & 360.7 & 235.3 & 56.1 \\
\hline $\mathrm{Sb}$ & $<0.1$ & $<0.1$ & $<0.1$ & $<0.1$ & $<0.1$ & $<0.1$ & $<0.1$ & $<0.1$ & $<0.1$ & $<0.1$ \\
\hline Sc & 19.0 & 11.0 & 26.0 & 7.0 & 7.0 & 6.0 & 16.0 & 30.0 & 18.0 & 6.0 \\
\hline Se & 0.6 & 0.8 & 0.7 & 0.6 & $<0.5$ & $<0.5$ & 0.5 & 1.2 & $<0.5$ & $<0.5$ \\
\hline $\mathrm{Sm}$ & 8.48 & 4.81 & 32.48 & 4.48 & 8.38 & 3.20 & 6.22 & 39.52 & 9.75 & 3.48 \\
\hline Sn & 14.89 & 8.51 & 5.32 & 2.13 & 3.19 & 20.36 & 10.63 & 5.32 & 9.57 & 3.19 \\
\hline Sr & 71.5 & 42.4 & 30.0 & 39.6 & 92.9 & 18.1 & 45.6 & 30.3 & 69.0 & 18.6 \\
\hline Ta & 47.38 & 52.62 & 55.77 & 26.21 & 359.13 & 21.38 & 24.11 & 174.85 & 23.27 & 41.93 \\
\hline $\mathbf{T b}$ & 0.94 & 0.52 & 2.17 & 0.47 & 0.86 & 0.39 & 0.67 & 2.54 & 0.97 & 0.39 \\
\hline Th & 16.7 & 16.1 & 10.1 & 7.7 & 3.3 & 4.1 & 14.8 & 10.1 & 16.0 & 4.3 \\
\hline $\mathrm{Ti}$ & 1.0 & 0.2 & 1.9 & 0.1 & $<0.1$ & $<0.1$ & 0.4 & 2.2 & 1.1 & $<0.1$ \\
\hline Tm & 0.48 & 0.22 & 0.59 & 0.15 & 0.23 & 0.10 & 0.33 & 0.76 & 0.40 & 0.10 \\
\hline $\mathbf{U}$ & 4.5 & 5.0 & 5.1 & 3.7 & 17.3 & 6.5 & 3.7 & 6.1 & 3.9 & 6.2 \\
\hline
\end{tabular}


Asia Pacific Journal of Energy and Environment, Volume 1, No 1 (2014)

\begin{tabular}{|c|c|c|c|c|c|c|c|c|c|c|}
\hline $\mathbf{V}$ & 153.0 & 159.0 & 156.0 & 84.0 & 33.0 & 54.0 & 135.0 & 171.0 & 141.0 & 51.0 \\
\hline $\mathbf{W}$ & 1.7 & 2.0 & 1.6 & 1.0 & 4.6 & 2.6 & 2.7 & 1.2 & 1.9 & 2.4 \\
\hline $\mathbf{Y}$ & 28.1 & 13.3 & 46.4 & 9.7 & 14.4 & 7.8 & 19.5 & 55.3 & 26.5 & 7.9 \\
\hline $\mathbf{Y b}$ & 2.93 & 1.35 & 3.74 & 0.88 & 1.94 & 0.67 & 2.09 & 4.44 & 2.53 & 0.59 \\
\hline $\mathbf{Z n}$ & 24.0 & 14.0 & 129.0 & 8.0 & 7.0 & 10.0 & 20.0 & 145.0 & 30.0 & 10.0 \\
\hline $\mathbf{Z r}$ & 488.2 & 278.0 & 195.5 & 148.1 & 401.0 & 64.2 & 288.2 & 184.1 & 388.9 & 60.2 \\
\hline
\end{tabular}

Table 7: Range and average values of trace elements in the whole rock and eluvial soils in some pits around the Oro pegmatites in (ppm)

\begin{tabular}{|c|c|c|c|c|}
\hline Trace elements & \multicolumn{2}{|c|}{ Whole rock pegmatite } & \multicolumn{2}{c|}{ Eluvial soil } \\
\hline & \multicolumn{2}{|c|}{$\mathrm{n}=10$} & \multicolumn{2}{c|}{$\mathrm{n}=18$} \\
\hline & Range & Average & Range & Average \\
\hline $\mathrm{Ta}$ & $0.21-200.21$ & 23.48 & $3.56-359.13$ & 63.98 \\
\hline $\mathrm{Cs}$ & $0.1-7.1$ & 2.90 & $5.0-116.9$ & 29.29 \\
\hline $\mathrm{Rb}$ & $4.1-383.6$ & 73.92 & $56.1-442.8$ & 207.19 \\
\hline $\mathrm{Sn}$ & $<1.06-14.89$ & 6.06 & $2.13-20.36$ & 8.10 \\
\hline $\mathrm{Nb}$ & $1.15-99.65$ & 29.30 & $34.32-972.05$ & 119.34 \\
\hline $\mathrm{Li}$ & $<20-43$ & 21.60 & $<20.0-102.0$ & 48.44 \\
\hline $\mathrm{Sr}$ & $3.8-156.3$ & 26.31 & $18.1-570.2$ & 107.74 \\
\hline $\mathrm{Y}$ & $0.7-18.9$ & 7.71 & $7.8-71.2$ & 27.53 \\
\hline $\mathrm{Ba}$ & $19-143$ & 59 & $63.0-3022.0$ & 626.19 \\
\hline $\mathrm{W}$ & $<0.5-0.9$ & 0.57 & $1.0-4.6$ & 1.84 \\
\hline $\mathrm{Be}$ & $<1-25$ & 4.70 & $3.0-381.0$ & 63.67 \\
\hline $\mathrm{Zr}$ & $4.7-459.1$ & 164.02 & $60.2-488.2$ & 255.71 \\
\hline $\mathrm{Ga}$ & $0.6-21.4$ & 10.76 & $23.9-43.8$ & 33.78 \\
\hline
\end{tabular}

Ginsburg et al., (1979) Ginsburg (1984) started to classify pegmatites based on the orogenrelated association, it is only the rare-element pegmatite class that includes tantalum deposits. The abyssal and muscovite classes have no significant tantalum mineralization; the miarolitic class, as originally characterized by Ginsburg et al., (1979) and as directly redefined by London (1986) has the potential, but so far no well-defined examples of economic Ta contents are known.

Most pegmatite types of the rare-element class may contain significant tantalum mineralization. In accordance with the classification developed by Beus (1960), Rudenko et al. (1975) and Cerny (1988), the pertinent pegmatite types and subtypes and subtypes can be categorized for the purpose of characterizing tantalum mineralization as follows;

(i) Beryl type, with beryl-columbite and beryl-columbite-phosphate subtypes;

(ii) Complex type, including spodumene, pentalite and amblygonite subtypes;

(iii) Complex type, lepidolite subtype;

(iv) Albite-spodumene type; and

(v) Albite type.

The criteria for the classification are bulk chemistry, geochemical signature of accessory minerals, internal structure and pressure/temperature conditions of crystallization (Cerny, 1991). Classification can also be based on the molecular proportions of $\mathrm{Al}_{2} \mathrm{O}_{3} /\left(\mathrm{CaO}+\mathrm{Na}_{2} \mathrm{O}+\mathrm{K}_{2} \mathrm{O}\right)$ allows peralkaline $(\mathrm{A}<\mathrm{NK})$, metaluminous $(\mathrm{CNK}>\mathrm{A}>\mathrm{NK})$ and peraluminous $(\mathrm{A}>\mathrm{CNK})$ varieties to be recognized.

From field evidence, bulk chemistry signatures and the $\mathrm{Ta} /(\mathrm{Ta}+\mathrm{Nb})$ versus $\mathrm{Mn} /(\mathrm{Mn}+\mathrm{Fe})$ plot, the Oro pegmatite is a complex pegmatite, of the rare element class and displays typical characteristic of the Lithium, Cesium and Tantalite (LTC) family. Apart from the silicic and peraluminous $\{\mathrm{A} / \mathrm{CNK}>1\}$ where $\mathrm{A}: \mathrm{Al}_{2} \mathrm{O}_{3}, \mathrm{CNK}:\left(\mathrm{CaO}+\mathrm{Na}_{2} \mathrm{O}+\mathrm{K}_{2} \mathrm{O}\right)$ character supports this ascertion (Cerny, 1991b; London 2005). LCT pegmatites as in this study are also known to contain moderate to abundant $\mathrm{Ta}-\mathrm{Nb}$ mineralization, gemstones and industrial minerals (Cerny 1989). 
Asia Pacific Journal of Energy and Environment, Volume 1, No 1 (2014)

Rare metal granitic pegmatites are products extreme differentiation of large reservoir of granitic magma. The proportion of rare metals in such pegmatites is an indication of the level of fractionation achieved in the final stages of granitic differentiation (Cerny, 1991). The geochemical characteristics of these rock suites are determined through the use of variation diagrams generated from the plots of certain elemental ratios of some selected elements. The following relationships are used in the plotting of the variation diagrams used in this study; $K / R b$ vs $C s, T a$ vs $\mathrm{Ga}, K / R b$ vs $R b$, $\mathrm{Na}_{2} \mathrm{O} / \mathrm{Al}_{2} \mathrm{O}_{3}$ vs $\mathrm{K}_{2} \mathrm{O} / \mathrm{Al}_{2} \mathrm{O}_{3}$, Ta vs Cs, Ta vs $\mathrm{Rb}$, Ta vs $(\mathrm{Cs}+\mathrm{Rb})$, Ta vs $\mathrm{Nb}$, Ta vs $\mathrm{K} / \mathrm{Cs}, \mathrm{Ta} / \mathrm{W}$ vs Cs, $\mathrm{Rb}$ vs $(\mathrm{Y}+\mathrm{Nb}), \mathrm{Zr}$ vs $\mathrm{SiO}_{2}$, Sr vs $\mathrm{Rb}$, $\mathrm{Rb}$ vs $\mathrm{Sr}$ and $\mathrm{Ta} /(\mathrm{Ta}+\mathrm{Nb})$ vs $\mathrm{Mn} /(\mathrm{Mn}+\mathrm{Fe})$.

Table 8: Elemental ratios of some selected elements of whole rock pegmatites of Oro

\begin{tabular}{|c|c|c|c|c|c|c|c|c|c|c|}
\hline Elemental ratios & RS1 & RS2 & RS3 & RS4 & RS5 & RS6 & RS7 & RS8 & RS9 & RS10 \\
\hline $\mathrm{Ba} / \mathrm{Rb}$ & 9.30 & 1.30 & 4.30 & 4.70 & 0.07 & 0.60 & 0.18 & 0.26 & 3.95 & 15.79 \\
\hline $\mathrm{K} / \mathrm{Ce}$ & 7.18 & 5.24 & 30.74 & 23.81 & 389.47 & 76.92 & 1640 & 397.79 & 39.56 & 3.92 \\
\hline $\mathrm{K} / \mathrm{Cs}$ & 8000 & 2666.7 & 1583.3 & 1636.4 & 1042.3 & 1090.9 & 1366.7 & 1309.1 & 1142.9 & 1600 \\
\hline $\mathrm{K} / \mathrm{Nb}$ & 695.7 & 439.6 & 114.1 & 118.6 & 74.3 & 134.1 & 184.1 & 201.3 & 79.2 & 404.0 \\
\hline $\mathrm{K} / \mathrm{Rb}$ & 195.1 & 148.2 & 128.4 & 133.3 & 19.3 & 55.9 & 80.1 & 82.2 & 88.4 & 140.4 \\
\hline $\mathrm{K} 2 \mathrm{O} / \mathrm{Na} 2 \mathrm{O}$ & 4.5 & 4.5 & 11.5 & 11 & 5.9 & 0.12 & 0.15 & 0.15 & 0.12 & 4.5 \\
\hline $\mathrm{Mg} / \mathrm{Li}$ & 3.16 & 6.32 & 15.79 & 15.79 & 15.79 & 169.52 & 22.11 & 18.95 & 185.12 & 9.47 \\
\hline $\mathrm{Na} / \mathrm{K}$ & 0.25 & 0.25 & 0.11 & 0.11 & 0.15 & 7.46 & 6.17 & 6.04 & 7.34 & 0.25 \\
\hline $\mathrm{Nb} / \mathrm{Ta}$ & 5.48 & 8.67 & 13.22 & 12.05 & 0.50 & 3.49 & 8.50 & 7.42 & 3.71 & 4.71 \\
\hline $\mathrm{Rb} / \mathrm{Ce}$ & 0.04 & 0.04 & 0.24 & 0.18 & 20.19 & 1.38 & 20.48 & 4.84 & 0.45 & 0.03 \\
\hline $\mathrm{Rb} / \mathrm{Cs}$ & 41 & 18 & 12.3 & 12.3 & 54.0 & 19.5 & 17.1 & 15.9 & 12.9 & 11.4 \\
\hline $\mathrm{Rb} / \mathrm{Sr}$ & 0.46 & 0.23 & 36.10 & 3.55 & 24.59 & 1.43 & 17.36 & 14.10 & 0.23 & 0.22 \\
\hline $\mathrm{Sr} / \mathrm{Rb}$ & 2.17 & 4.37 & 0.28 & 0.28 & 0.04 & 0.70 & 0.06 & 0.07 & 4.32 & 4.54 \\
\hline $\mathrm{Ta} / \mathrm{W}$ & 0.53 & 0.53 & 3.15 & 3.15 & 222.50 & 12.84 & 10.48 & 8.03 & 12.11 & 1.05 \\
\hline $\mathrm{Zr} / \mathrm{Hf}$ & 15.67 & 33.33 & 38.38 & 36.49 & 20.73 & 40.58 & 18.06 & 21.86 & 38.26 & 47.00 \\
\hline $\mathrm{Zr} / \mathrm{Sn}$ & 1.47 & 9.52 & 369.34 & 138.78 & 1.53 & 11.45 & 3.06 & 1.60 & 53.95 & 17.91 \\
\hline $\mathrm{Na} 2 \mathrm{O} / \mathrm{Al} 2 \mathrm{O} 3$ & 0.03 & 0.02 & 0.01 & 0.01 & 0.03 & 0.36 & 0.45 & 0.44 & 0.24 & 0.02 \\
\hline $\mathrm{K} 2 \mathrm{O} / \mathrm{Al} 2 \mathrm{O} 3$ & 0.12 & 0.08 & 0.11 & 0.11 & 0.16 & 0.04 & 0.07 & 0.07 & 0.03 & 0.07 \\
\hline $\mathrm{Y}+\mathrm{Nb})$ & 1.85 & 3.22 & 35.56 & 27.28 & 102.25 & 42.8 & 57.24 & 40.46 & 55.92 & 3.48 \\
\hline $\mathrm{Ta} / \mathrm{Ta}+\mathrm{Nb})$ & 0.15 & 0.10 & 0.07 & 0.08 & 0.67 & 0.22 & 0.11 & 0.12 & 0.21 & 0.18 \\
\hline $\mathrm{Mn} / \mathrm{Mn}+\mathrm{Fe})$ & 0.06 & 0.03 & 0.02 & 0.02 & 0 & 0.01 & 0.04 & 0.04 & 0.01 & 0.04 \\
\hline $\mathrm{A} / \mathrm{CNK}$ & 6.64 & 9.83 & 7.96 & 7.73 & 5.43 & 2.29 & 1.80 & 1.82 & 3.39 & 9.69 \\
\hline
\end{tabular}

Table 9: Elemental ratios of some selected elements of eluvial soils of Oro

\begin{tabular}{|c|c|c|c|c|c|c|c|c|c|c|}
\hline Elemental ratios & SS1 & SS2 & SS3 & SS4 & SS5 & SS6 & SS7 & SS8 & SS9 & SS10 \\
\hline $\mathrm{Ba} / \mathrm{Rb}$ & 0.89 & 0.97 & 1.80 & 1.14 & 0.68 & 1.08 & 0.78 & 2.11 & 1.10 & 1.12 \\
\hline $\mathrm{K} / \mathrm{Ce}$ & 58.59 & 108.11 & 121.31 & 74.79 & 84.53 & 98.21 & 72.91 & 103.20 & 54.52 & 100.00 \\
\hline $\mathrm{K} / \mathrm{Cs}$ & 393.81 & 378.38 & 212.89 & 454.55 & 328.67 & 423.08 & 362.75 & 218.95 & 340.25 & 440.00 \\
\hline $\mathrm{K} / \mathrm{Nb}$ & 96.33 & 40.46 & 118.88 & 87.28 & 4.84 & 81.82 & 104.80 & 293.61 & 116.68 & 51.09 \\
\hline $\mathrm{K} / \mathrm{Rb}$ & 34.01 & 32.54 & 41.74 & 39.46 & 34.51 & 37.94 & 31.75 & 47.41 & 34.85 & 39.22 \\
\hline $\mathrm{K} 2 \mathrm{O} / \mathrm{Na} 2 \mathrm{O}$ & 21.40 & 33.50 & 26.14 & 0.42 & 0.56 & 0.26 & 22.25 & 34.33 & 24.75 & 0.26 \\
\hline $\mathrm{Mg} / \mathrm{Li}$ & 23.53 & 30.44 & 176.14 & 21.05 & 26.32 & 47.37 & 21.57 & 229.63 & 41.51 & 34.62 \\
\hline $\mathrm{Na} / \mathrm{K}$ & 0.05 & 0.04 & 0.03 & 0 & 0 & 0 & 0.04 & 0.03 & 0.04 & 0 \\
\hline $\mathrm{Nb} / \mathrm{Ta}$ & 1.95 & 2.63 & 2.29 & 1.53 & 2.71 & 1.26 & 2.93 & 0.33 & 3.02 & 1.03 \\
\hline $\mathrm{Rb} / \mathrm{Ce}$ & 1.72 & 3.30 & 2.91 & 1.90 & 2.45 & 2.60 & 2.30 & 2.18 & 1.57 & 2.55 \\
\hline $\mathrm{Rb} / \mathrm{Cs}$ & 11.58 & 11.63 & 5.10 & 11.52 & 9.52 & 11.21 & 11.43 & 4.62 & 9.76 & 11.22 \\
\hline $\mathrm{Rb} / \mathrm{Sr}$ & 3.66 & 4.01 & 12.14 & 2.24 & 1.47 & 3.22 & 5.11 & 11.90 & 3.41 & 3.02 \\
\hline $\mathrm{Sr} / \mathrm{Rb}$ & 0.27 & 0.25 & 0.08 & 0.45 & 0.68 & 0.30 & 0.20 & 0.08 & 0.29 & 0.33 \\
\hline $\mathrm{Ta} / \mathrm{W}$ & 27.87 & 26.31 & 34.86 & 26.21 & 78.07 & 8.22 & 8.93 & 145.71 & 12.25 & 17.47 \\
\hline $\mathrm{Zr} / \mathrm{Hf}$ & 28.90 & 37.57 & 31.03 & 36.12 & 16.85 & 22.14 & 31.70 & 29.69 & 33.82 & 23.15 \\
\hline $\mathrm{Zr} / \mathrm{Sn}$ & 32.80 & 32.67 & 36.75 & 69.53 & 125.71 & 3.15 & 27.14 & 34.61 & 40.60 & 18.87 \\
\hline $\mathrm{Na} 2 \mathrm{O} / \mathrm{Al} 2 \mathrm{O} 3$ & 0.002 & 0.001 & 0.003 & 0 & 0 & 0 & 0.002 & 0.003 & 0.002 & 0 \\
\hline $\mathrm{K} 2 \mathrm{O} / \mathrm{Al} 2 \mathrm{O} 3$ & 0.04 & 0.03 & 0.08 & 0.02 & 0.02 & 0.01 & 0.03 & 0.09 & 0.04 & 0.01 \\
\hline $\mathrm{(Y+Nb)}$ & 120.49 & 151.72 & 171.26 & 49.8 & 986.45 & 34.69 & 90.11 & 113.54 & 96.78 & 50.96 \\
\hline $\mathrm{Ta} / \mathrm{Ta}+\mathrm{Nb})$ & 0.34 & 0.28 & 0.31 & 0.40 & 0.27 & 0.44 & 0.26 & 0.75 & 0.25 & 0.49 \\
\hline $\mathrm{Mn} / \mathrm{Mn}+\mathrm{Fe})$ & 0.02 & 0.003 & 0.01 & 0.01 & 0.01 & 0 & 0.01 & 0.01 & 0.01 & 0 \\
\hline
\end{tabular}


Considering the $\mathrm{K} / \mathrm{Rb}$ versus $\mathrm{Rb}$ variation plot for the Oro whole rock pegmatites samples and the eluvial soil samples reveals a consistent trend (Fig.5), indicating the mineralization in the pegmatite of this study area to be high (Staurov et al., 1969). These plots also show a conspicuous distribution pattern of separation in the Oro pegmatite along the differentiation trend of the pegmatite in the study area. The pegmatites show a high differentiation and plot within the field of mineralization (Staurov et al., 1969).

Fig.4: $\mathrm{K} / \mathrm{Rb}$ vs $\mathrm{Rb}$ distribution pattern in the whole rock and eluvial soils of Oro pegmatite

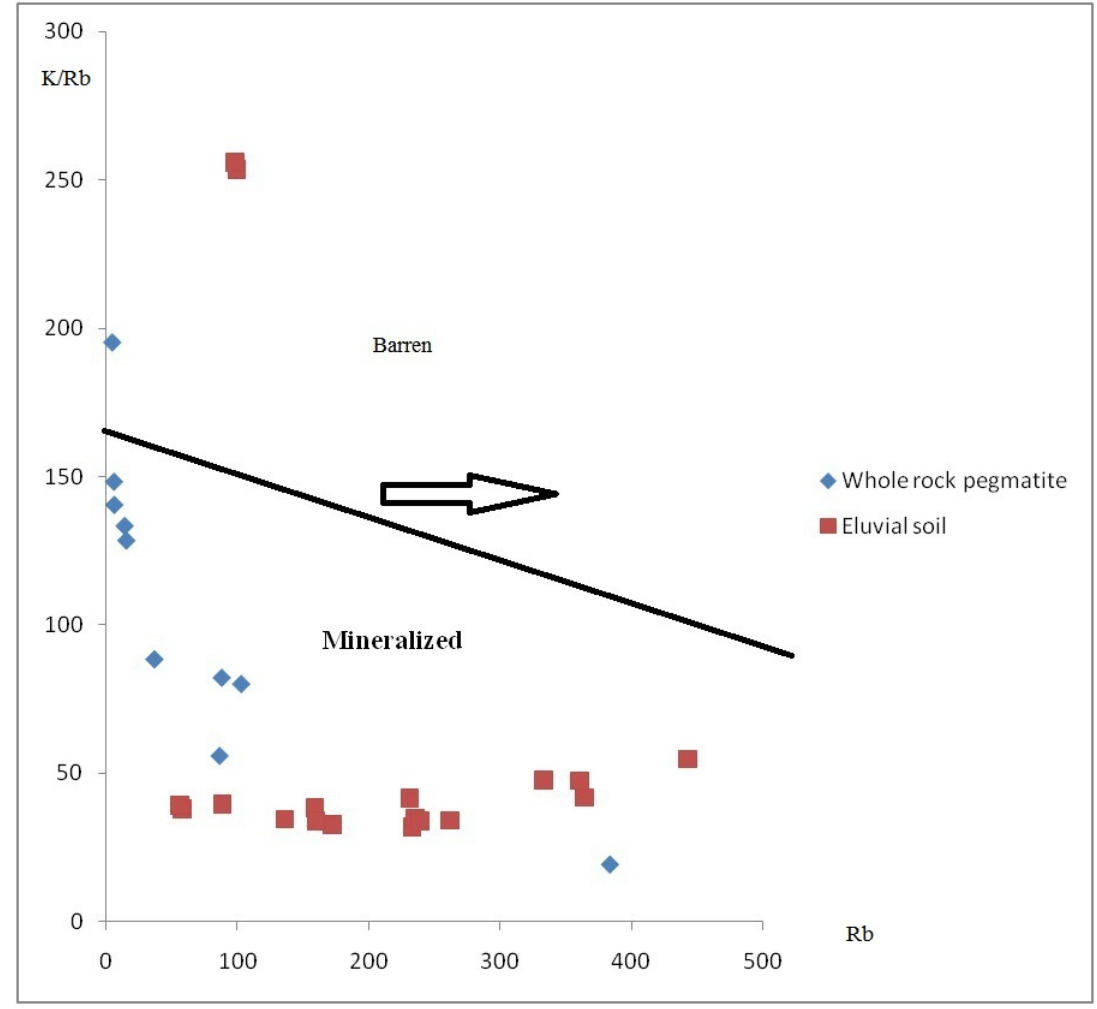

Arrow indicate normal differentiation trend after Staurov et al., (1969)

Also considering the variation plots of Ta versus $\mathrm{Ga}$, Ta versus Cs, Ta versus $\mathrm{Rb}$, Ta versus $(\mathrm{Cs}+\mathrm{Rb}$ ), Ta versus $\mathrm{Nb}$, Ta versus $\mathrm{K} / \mathrm{Cs}$ and $\mathrm{Ta} / \mathrm{W}$ versus $\mathrm{Cs}$ (Fig. 6,7,8,9,10,11 and 12), these plots also show the whole rock samples plot below the Beus line of mineralization, (1966) while the eluvial soil samples of the Oro pegmatites plotting above the mineralized line of Beus, (1966) and Gordiyenko, (1971), this is an indicative that the rare metals have broken down in the whole rock samples due to in situ weathering and have also migrated to the soils thereby making the soils more enriched in the rare metals than the whole rock samples itself.

The overall trends invariably indicate the relative enrichment of Ta in the soil samples. Ta concentration values in the pegmatite samples of the area of study fall below Egbe pegmatite (Matheis, 1987) as well as Ijero pegmatite (Okunlola and Jimba, 2006) and much below the highly productive Tanco, Homestead and Withrope Ta-pegmatites (Cerny, 1989; Beus, 1966). 
Asia Pacific Journal of Energy and Environment, Volume 1, No 1 (2014)

Fig.5: Plot of Ta vs Ga for the Oro Pegmatite.

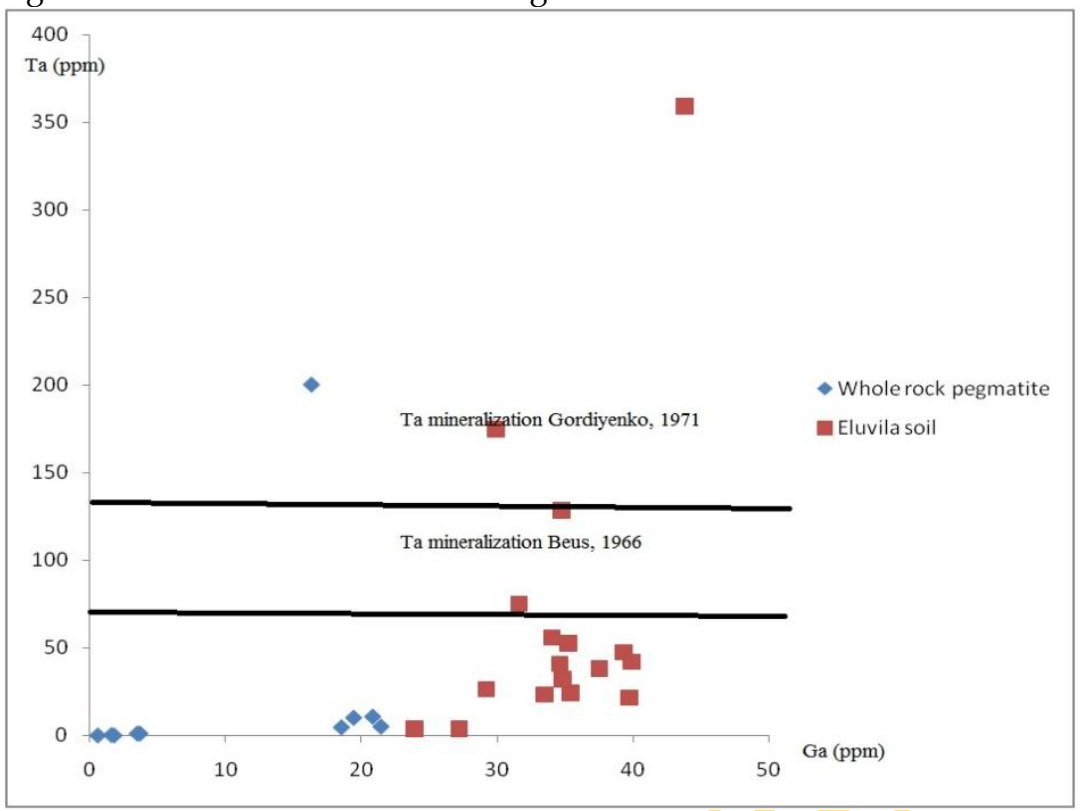

Fig.6: Plot of Ta vs Cs for the pegmatites of Oro study area

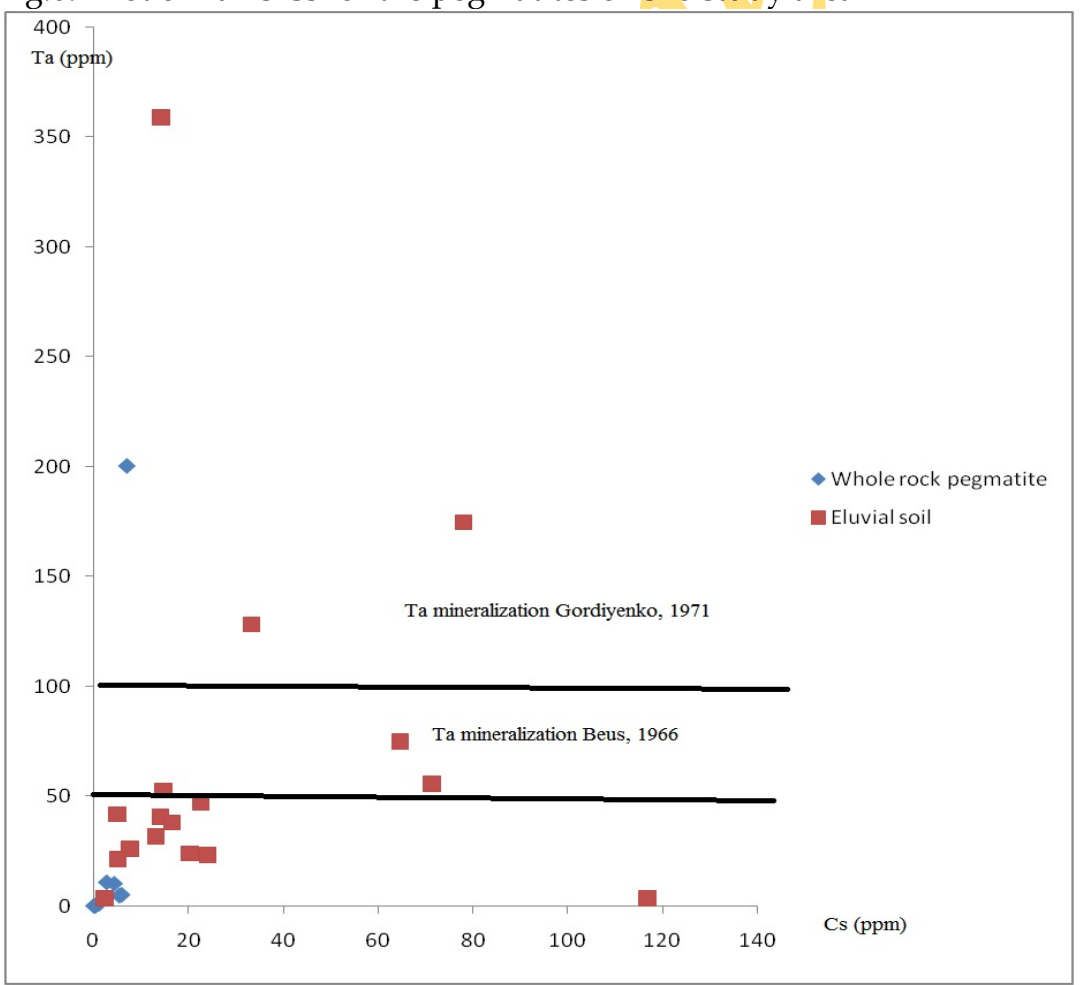

(after Moller and Morteani, 1987). 
Fig.7: Plot of Ta vs Rb for the pegmatites of Oro study area

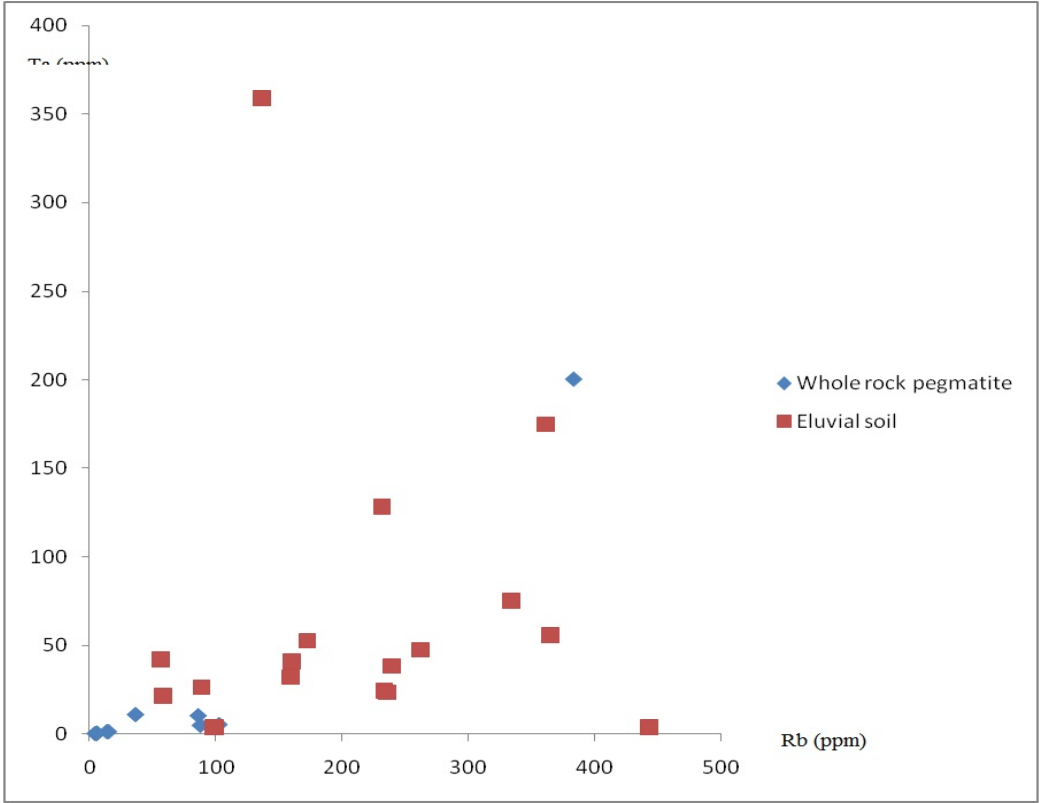

(after Moller and Morteani, 1987).

Fig.8: Plot of Ta vs $\mathrm{Cs}+\mathrm{Rb}$ for the pegmatites of Oro study area

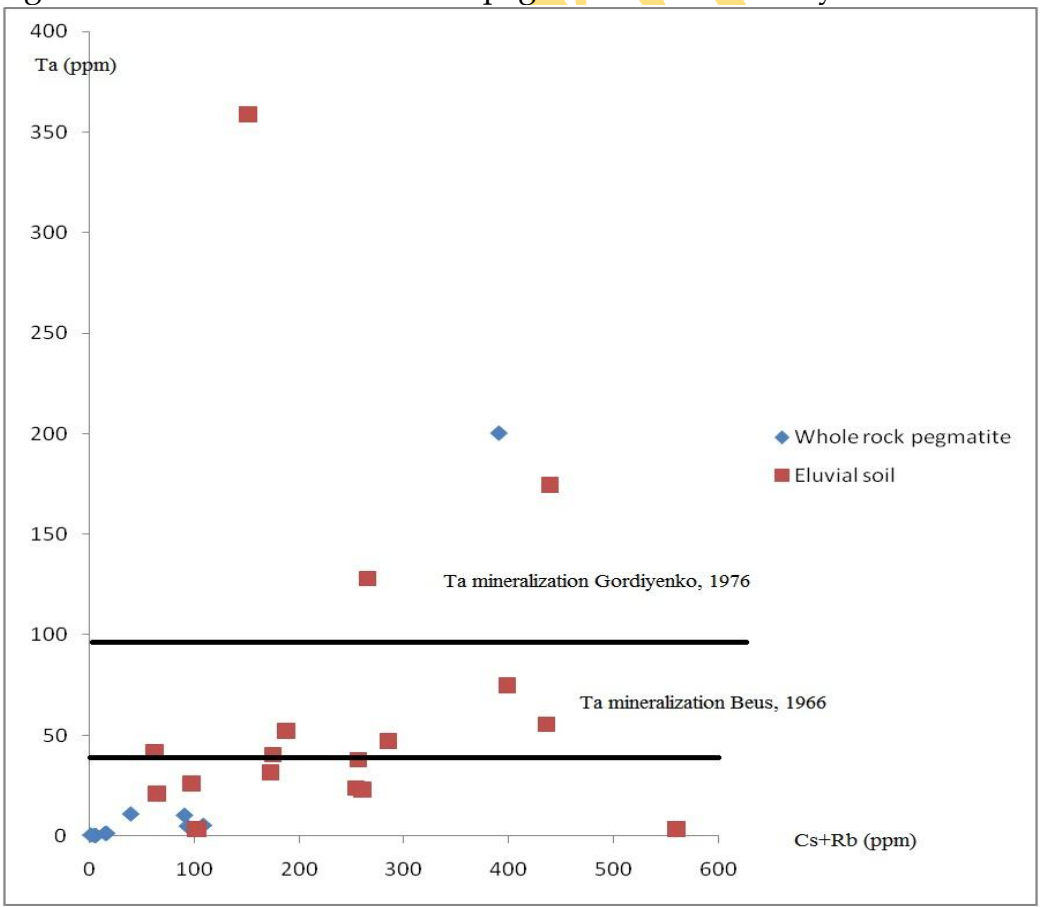

(after Gaupp et al., 1984). 
Fig.9: Plot of Ta vs K/Cs ratio for the pegmatites of Oro study area

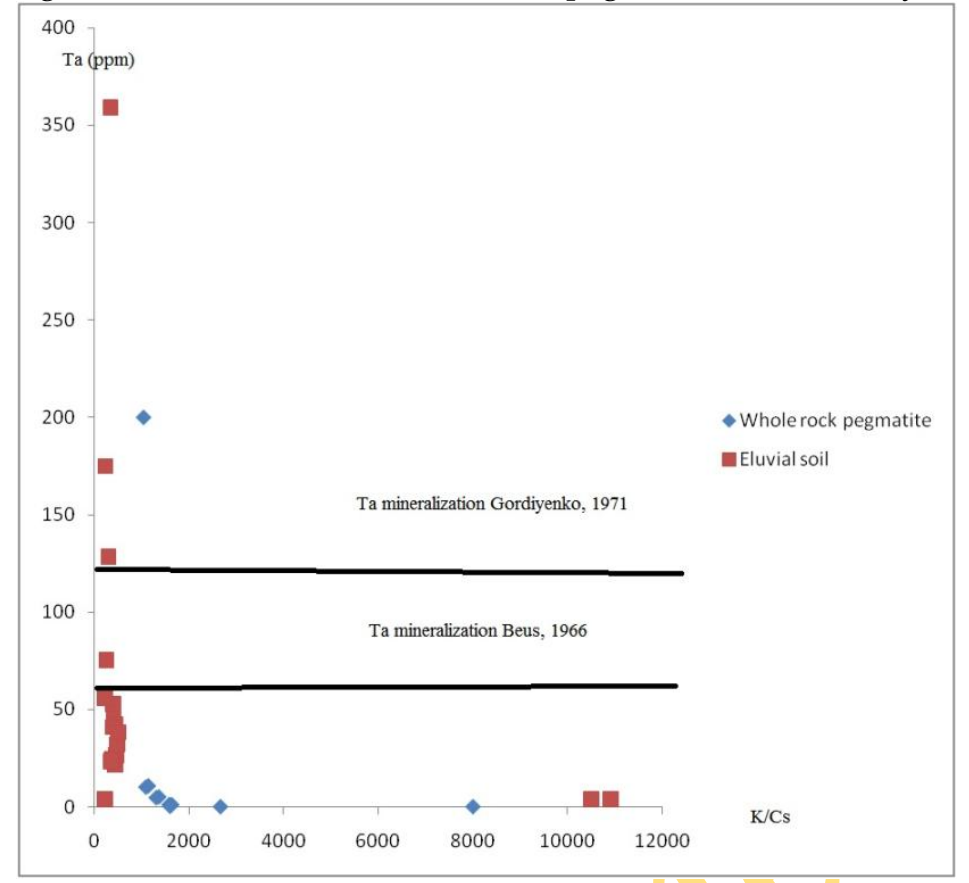

(after Gordiyenko, 1971 and Beus, 1966).

The trend of compositional variation in the columbite-tantalite quadrilateral has direct relationship with degree of fractionation. Pegmatites whose values plot in the field ferro-columbite have been generally interpreted as primitive and not good sources of $\mathrm{Ta}$ ores (Breaks et al., 1999). Most data here plot in the field of ferrocolumbite and only one

plot in the field of ferro-tantalite. This indicates that the pegmatites of Oro area are moderately promising for tantalite and other rare metals. These $\mathrm{Rb} / \mathrm{Y}+\mathrm{Nb}$ plot (Fig.10)

- shows that the pegmatites plot in the field of the volcanic arc granite with few of the samples plotting clearly within plate granite.

Fig 10: $\mathrm{Rb}$ vs $(\mathrm{Y}+\mathrm{Nb})$ discriminant diagram for the whole rock pegmatites and eluvial soils of Oro (after Pearce et al., 1984)

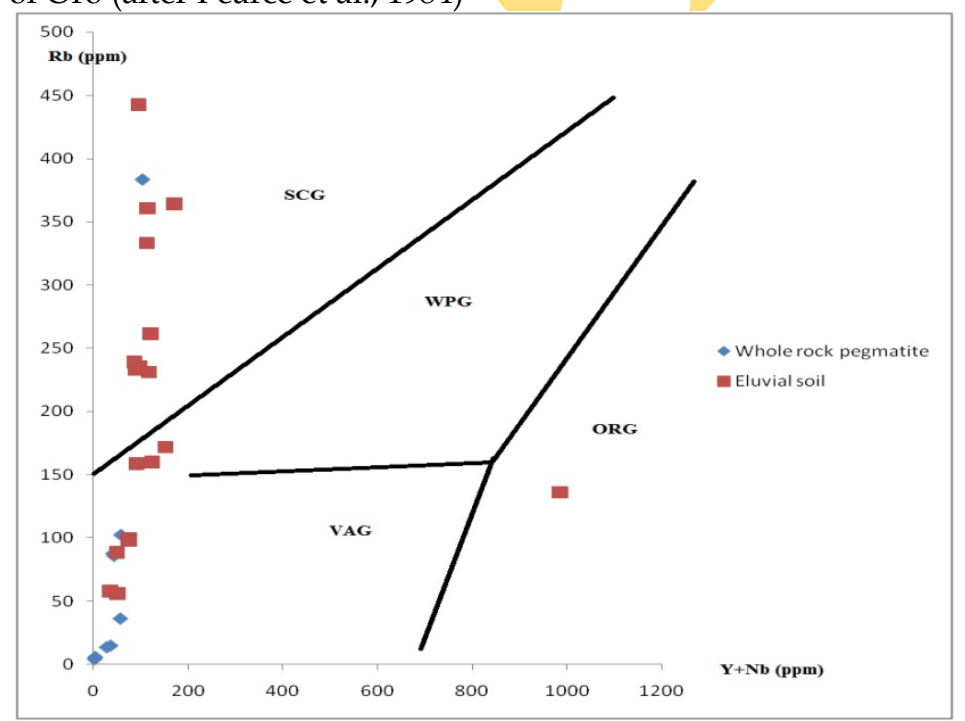

VAG-Volcanic Arc Granite SCG-Syn-Collisional Granite WPG- Within Plate Granite ORG- Oceanic Ridge Granite

The variation plot of $\mathrm{Rb}$ versus $\mathrm{Sr}$ (ppm) reveals the crustal thickness during emplacement of these pegmatite bodies (Fig.13) which reached about $30 \mathrm{~km}$ as shown from the $\mathrm{Rb} / \mathrm{Sr}$ plot of Condie, (1976). 
Asia Pacific Journal of Energy and Environment, Volume 1, No 1 (2014)

Fig.11: Plot of Rb-Sr for the pegmatites of Oro study area. ( after Condie, 1976).

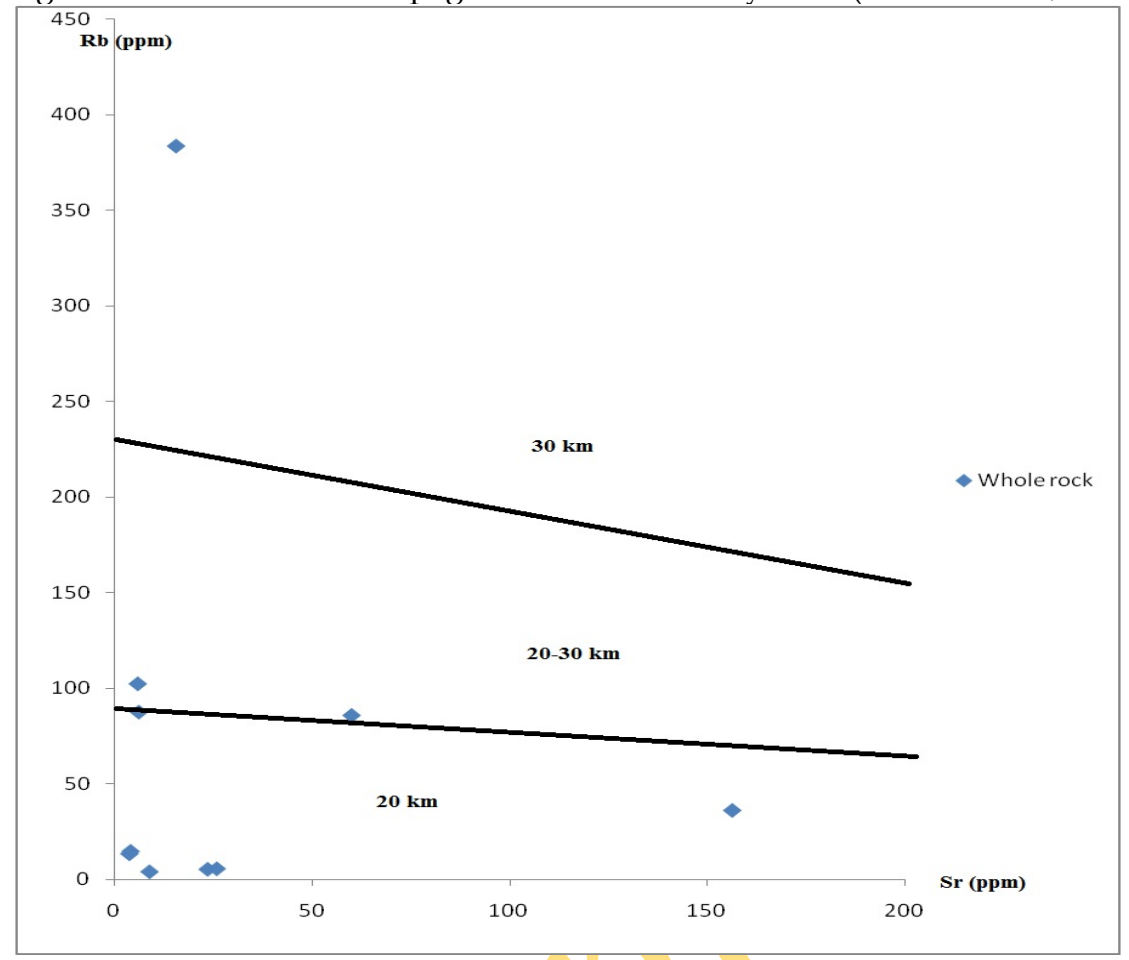

Fig.12: Plot of $\mathrm{Na}_{2} \mathrm{O} / \mathrm{Al}_{2} \mathrm{O}_{3}$ vs $\mathrm{K}_{2} \mathrm{O} / \mathrm{Al}_{2} \mathrm{O}_{3}$ (Wt \%) showing variation diagram for the field of Igneous and Metasedimentary rocks of Oro pegmatites.

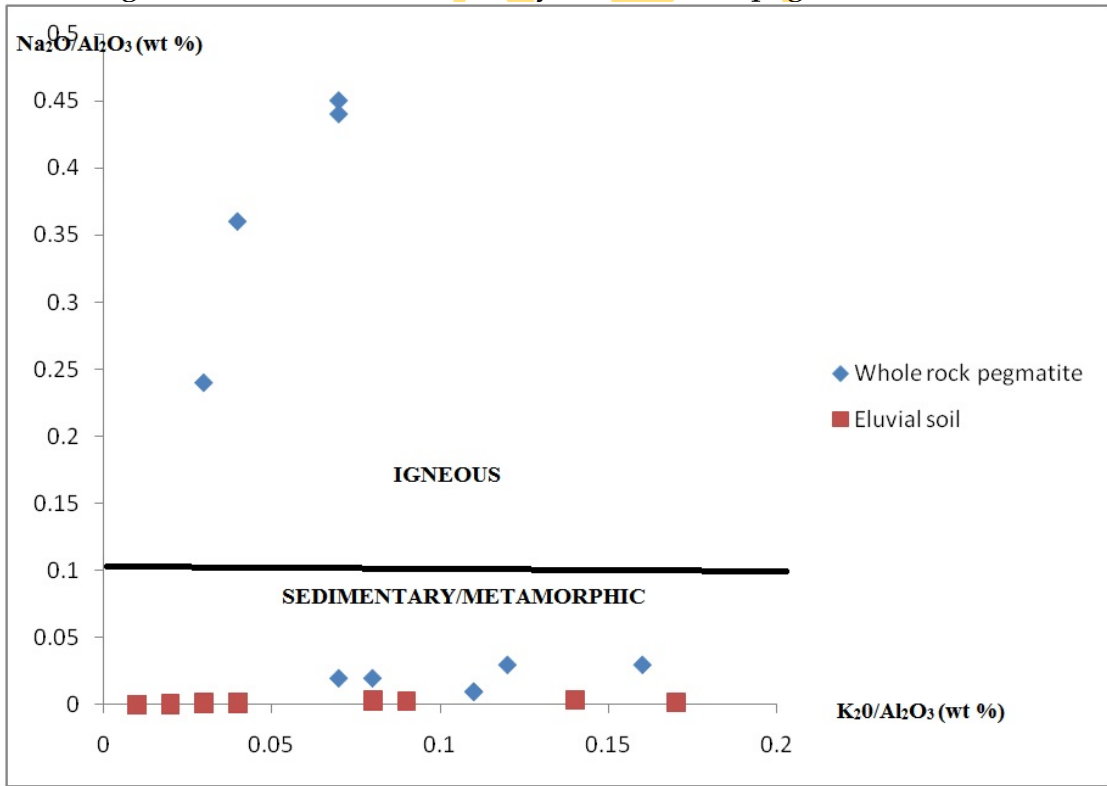


The degree of albitization is revealed by the triangular Ti-Sn-(Nb+Ta) discriminant plot which plots in the zone of albitization (Fig. 12) for the Oro pegmatites. This plot also reveals a high degree of albitization and it indicates a significant difference between the mineralized and unmineralized pegmatite samples (Matheis and Emofurieta, 1990; Okunlola and King, 2003; Okunlola and Somorin, 2005; Jacobson and Webb, 1946), however these values are still low compared to those of the economically viable bodies like Tanco Canada (Moller and Morteani, 1987), but there are indications that the pegmatites of Oro area compare favorably with those of other mineralized pegmatite areas like Egbe and Igbeti areas southwestern Nigeria (Matheis and Emofurieta, 1987; Okunlola and Oyedokun, 2009). This is also indicative of the degree of fractionation.

Fig. 13: Triangular Ti-Sn-(Nb+Ta) Plot for Oro pegmatites (after Kuster, 1990)

\section{DEGREE OF ALBITIZATION}

$$
\mathrm{Ti}-\mathrm{Sn}-\mathrm{Nb}+\mathrm{Ta}(\mathrm{ppm})
$$

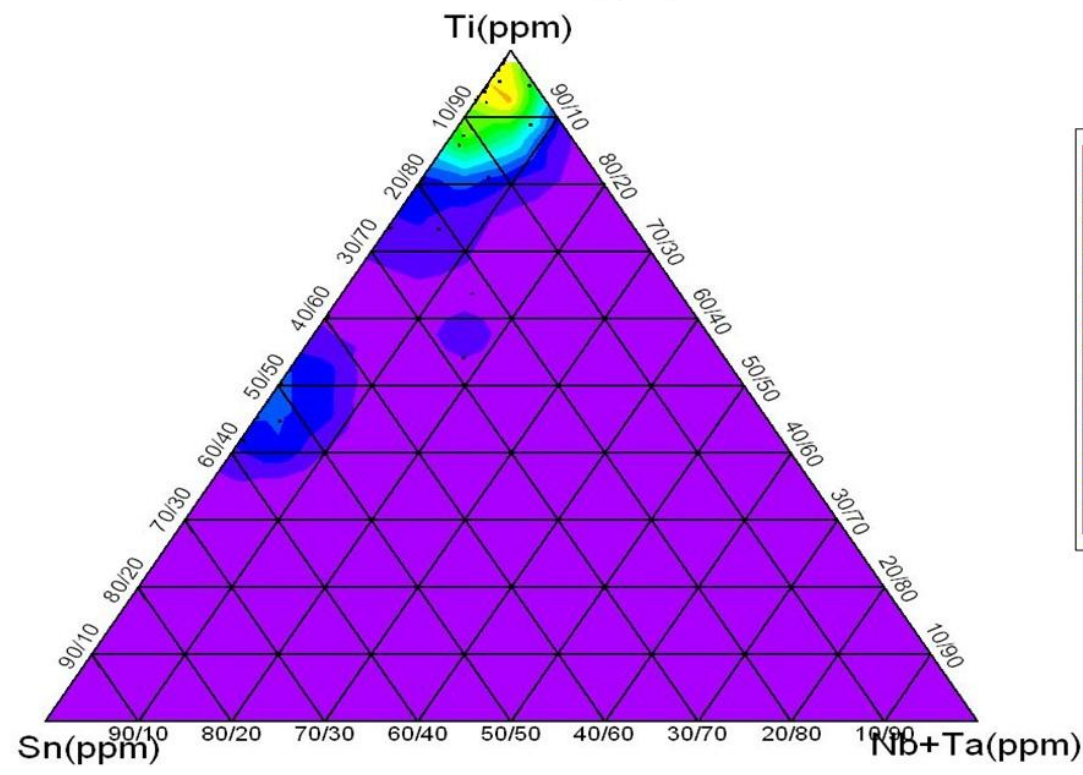

The Chondrite normalized plot (Fig.13) of the rare earth elements shows high light REE (LREE) (Ce, Nd) values and low heavy (HREE) (Tb, Dy, Ho, Er, Tm, Lu and Yb). There is a weak negative Europium (Eu) anomaly and a strong positive Ce anomaly. This is especially characteristic of LCT pegmatites with attendant high fractionation. (Cerny, 1991b) However, Taylor et al., (1986) had suggested earlier that where there is a weak negative Ce signature and a strong negative Eu signature as in this case of Oro pegmatite samples it is an evidence of considerable fractionation and metasomatism. Also, Piper, (1974) and Garba, (2003) believe that Negative Ce signature of rare metal pegmatite is taken to indicate oxidizing condition during mineralization and interaction between magmatic, melt fluids and host rocks over long distance sometimes. 
Table 12: REE/Chondrite normalized data for Oro pegmatite

\begin{tabular}{|c|c|c|c|c|c|c|c|c|c|c|}
\hline REE & RS1 & RS2 & RS3 & RS4 & RS5 & RS6 & RS7 & RS8 & RS9 & RS10 \\
\hline $\mathrm{Y}$ & 1.372 & 2.74 & 37.04 & 23.72 & 5.10 & 13.72 & 24.89 & 9.21 & 30.38 & 2.94 \\
\hline $\mathrm{La}$ & 3.20 & 8.81 & 7.82 & 10.40 & 7.72 & 11.45 & 0.86 & 1.02 & 9.54 & 9.67 \\
\hline $\mathrm{Ce}$ & 98.03 & 134.38 & 54.38 & 66.53 & 16.72 & 54.91 & 4.4 & 15.93 & 71.19 & 179.52 \\
\hline $\mathrm{Pr}$ & 0.19 & 0.44 & 0.62 & 0.83 & 0.36 & 0.77 & 0.05 & 0.06 & 0.83 & 0.48 \\
\hline $\mathrm{Nd}$ & 4.92 & 7.2 & 12.42 & 17.4 & 6.06 & 14.94 & 1.02 & 0.84 & 19.02 & 7.56 \\
\hline $\mathrm{Sm}$ & 0.12 & 0.26 & 0.66 & 0.86 & 0.21 & 0.66 & 0.14 & 0.10 & 1.08 & 0.30 \\
\hline $\mathrm{Eu}$ & 0.007 & 0.02 & 0.03 & 0.03 & 0.02 & 0.04 & 0.002 & 0.002 & 0.09 & 0.02 \\
\hline $\mathrm{Gd}$ & 0.16 & 0.26 & 0.72 & 0.80 & 0.17 & 0.59 & 0.31 & 0.15 & 1.13 & 0.25 \\
\hline $\mathrm{Tb}$ & 0.002 & 0.0005 & 0.02 & 0.02 & 0.006 & 0.02 & 0.02 & 0.009 & 0.03 & 0.005 \\
\hline $\mathrm{Dy}$ & 0.17 & 0.45 & 3.17 & 2.63 & 0.67 & 1.80 & 2.44 & 1.01 & 3.86 & 0.50 \\
\hline $\mathrm{Ho}$ & 0.002 & 0.005 & 0.05 & 0.04 & 0.007 & 0.02 & 0.03 & 0.009 & 0.05 & 0.004 \\
\hline $\mathrm{Er}$ & 0.01 & 0.03 & 0.42 & 0.30 & 0.04 & 0.13 & 0.20 & 0.08 & 0.37 & 0.02 \\
\hline $\mathrm{Tm}$ & 0 & 0.0006 & 0.011 & 0.008 & 0.002 & 0.003 & 0.006 & 0.002 & 0.008 & 0.0006 \\
\hline $\mathrm{Yb}$ & 0.02 & 0.024 & 0.47 & 0.28 & 0.05 & 0.14 & 0.204 & 0.09 & 0.35 & 0.02 \\
\hline $\mathrm{Lu}$ & 0 & 0.0007 & 0.014 & 0.010 & 0.002 & 0.003 & 0.005 & 0.002 & 0.010 & 0.001 \\
\hline
\end{tabular}

Fig.14: REE chondrite normalized plots of the pegmatite samples

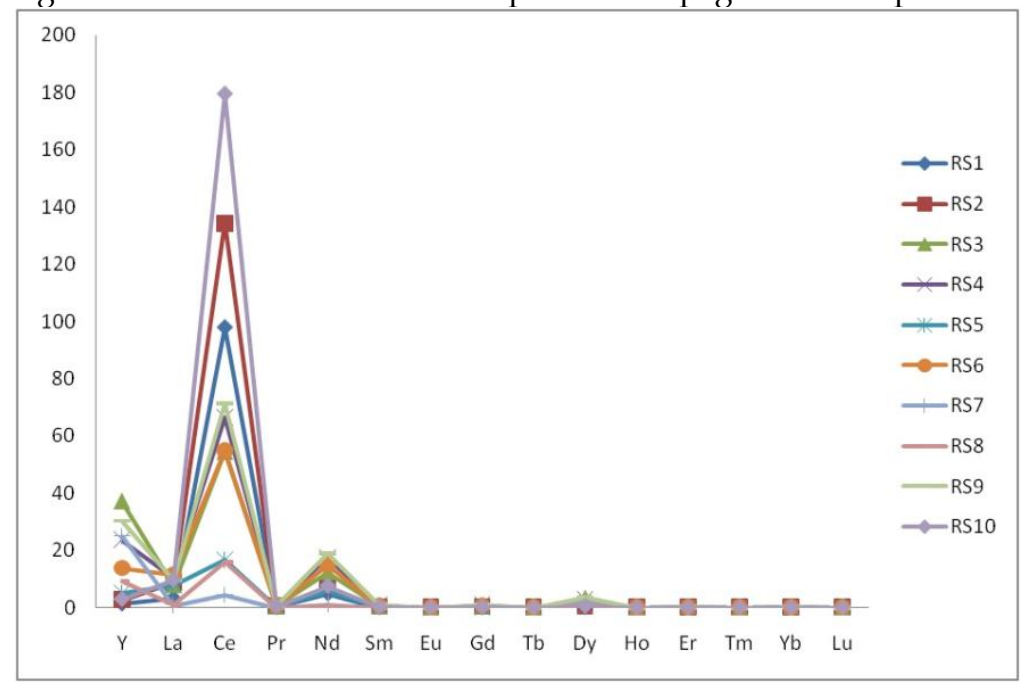

\section{Conclusions}

The pegmatites occur as vertically dipping and low-lying dykes, intruding the older quartz mica schist and granite gneiss. The pegmatites are observed to be rich in gem beryl, tourmaline, quartz and feldspar. Optical studies show the dominance of microcline, quartz, albite, biotite and muscovite with other opaques and accessory minerals.

Chemical data show that the pegmatites are highly siliceous, with silica contents ranging from $71.49-97.77 \%$. The alumina contents of the whole rock pegmatite range from 0.73$15.20 \%$. The $\mathrm{K} / \mathrm{Rb}$ ratios, in the range of 19.3-195.1ppm were obtained from the whole rock pegmatite samples of the area, while the $\mathrm{Ba} / \mathrm{Rb}$ ratio $(0.07-15.79 \mathrm{ppm})$ of the samples are comparable with other pegmatites from the southwestern Nigeria (Okunlola and Jimba, 2006). The relatively low average of $\mathrm{K} / \mathrm{Rb}(107.13 \mathrm{ppm})$ with $\mathrm{Rb} / \mathrm{Sr}(9.83 \mathrm{ppm})$ ratios of the pegmatites indicate a low to moderate degree of fractionation. 


\section{ACKNOWLEDGEMENTS}

I am greatly indebted to God, my dear wife Gbemisola Helen Oyebamiji for her love, care, moral support and encouragement and also to my parents, Mr. and Mrs. J. S. Oyebamiji, my Miss Olaitan Oyebamiji, Mrs. Abimbola Olalekan, my brother Mr. Olumuyiwa Oyebamiji for their financial support at all times, may God bless my family.

\section{REFERENCES}

[1] Adekeye, J.I.D. and Akintola, O.F., (2007). Geochemical features of rare-metal pegmatites in Nasarawa area, central Nigeria. Journal of Mining and Geology, Vol. 43, No. 1 pp. 15-21.

[2] Akintola, A. I., Ikhane, P. R., Laniyan, T. A., Akintola, G. O., Kehinde-Phillips, O. O. (2011). Compositional trends and rare-metal $(\mathrm{Ta}-\mathrm{Nb})$ mineralization potential of Precambrian pegmatites in Komu area, Southwestern Nigeria. International Journal of Current Research Vol.4 issue 02, pp. 031-039

[3] Akintola, A. I., Ikhane, P. R., Okunlola, O. A., Akintola, G. O., Oyebolu, O. O. (2012). Compositional features of Precambrian pegmatites of Ago-Iwoye area Southwestern, Nigeria. Journal of Ecology and the Natural Environment Vol. 4(3), pp.71-87

[4] Beus, A. A. (1960). Geochemistry of beryllium and the Genetic Types of Beryllium Deposits. Acad. Sci. USSR, Moscow, USSR (in Russ.; Engl. Translation: Freeman \& Co., San Francisco, California

[5] Beus, A.A. (1966). Distribution of tantalum and niobium in muscovites from granitic pegmatites.Geokhimiya, Vol.10, pp. 1216-1220.

[6] Breaks, F. W., Twindle, A. G and Smith, S. R. (1999). Rare metal mineralization associated with Berens River-Sachiago Subprovincial Boundary, Northwestern Ontario: Discovery of a new zone of complex-type, petalite sub-type of pegmatite and implications for future exploration. Ontario Geological Survey Miscellaneous paper 169,33p

[7] Cerny, P. (1989). Characteristics of pegmatite deposits of tantalum, In Moller, P., Cerny,P., and Saupe., F., (eds) Lanthanides,Tantalum and Niobium. Society For Geology Applied To Mineral Deposits, Special Publication 7; Springer Verlag, pp.192-236.

[8] Cerny, P. (1991a). Geochemical and petrogenetic features of mineralization in rare element granitic pegmatites in the light of current research. Applied Geochemistry; Vol.18, pp. 48-68.

[9] Cerny, P. (1991b). Rare-element granitic pegmatites-regional to global environments and Petrogenesis. Geo-Science Canada; Vol.18, pp. 49-62.

[10] Cerny, P. (1992). Geochemical and Structural evolution of micas in the Rozna and Dubra voda pegmatites, Czech Republic. Mineralogy And Petrology, Vol.52, pp 4-9.

[11] Condie, K.D. (1976). Trace element geochemistry of Archean granite rocks from the Barberton Region, South Africa, AM. Mineral. Earth Planet Sc. Letter, pp. 300-400.

[12] Ekwueme, B. N., (1985). Elementary Crystallography and Mineralogy (including Optics).

[13] Elueze, A.A., Itiola, O.A., and Nton, M.E. (2004). Preliminary investigation on industrial properties of the Olode-Falansa pegmatite, Southwestern Nigeria. Global Jour. Geol. Sci. 2(2), 255-264

[14] Garba, I. (2003). Geochemical discrimination of newly discovered rare metal bearing and barren Pegmatites in the Pan-African $(600+150 \mathrm{Ma})$ basement of northern Nigeria. Applied Earth Science Transaction Institute Of Mining And Mettallurgy 13: Vol.112 pp. B287-B291.

[15] Garrels, R.M. and Mackenzie, F.T. (1971). Evolution of Igneous and Sedimentary Rocks. W.W. Norton and Company, Inc. New York, N.Y. 394p.

[16] Ginsburg, A. I. (1984). The geological condition of the location and the formation of granitic pegmatites: $27^{\text {th }}$ IGC proceedings 15: $245-260$

[17] Ginsburg, A. I., Timofeyev, I. N., and Feldmann, L. G., (1979). Principles of geology of the granite pegmatites: Moscow, Nedra. 296p

[18] Gordiyenko, V.V. (1971). Concentration of $\mathrm{Li}, \mathrm{Rb}$, and Cs in potash feldspar and muscovite as criteria for assessing rare metal mineralization in granite pegmatites. Int. Geology Rev. Vol.13, 134-142.

[19] Jacobson, R.R.E. and Webbs, J.S. (1946). The Pegmatite of Central Nigeria. Geol. Surv. Nig. Bull, 17, 40-61.

[20] Kuster, D. (1990). Rare metal pegmatites of Wamba, central Nigeria their formation in relationship to late Pan-African granites. Mineral Deposita; Vol.25, pp. 25-28. 
[21] London, D. (1986a). Formation of tourmaline-rich gem pockets in miarolitic pegmatites. Am Mineral 71: 396-405

[22] London, D. (2005). Granitic pegmatites: An assessment of current concepts and directions for the Future LITHOS. Vol.8, pp. 281-303.

[23] Matheis, G. (1981). Trace element pattern in lateritic soils applied to geochemical exploration. Geochm. Exploration, 15, pp. 471-480

[24] Matheis, G. and Emofurieta, W.O. (1987). Nigerian Rare metal pegmatites and their lithological framework. J. Geol., 22, pp. 271-291.

[25] Matheis, G., Emofurieta, W. O. and Ohweriei, S. F., (1982). Trace element distribution in tin bearing pegmatites of Southwestern Nigeria. In Metallization. Associated with Acid Magmatism. Evans. M. (ed.), Wiley London. Pp. 205-220

[26] Moller, P and Morteani, G. (1987). Geochemical exploration guide for Tantalum pegmatites. Economic Geology, Vol.42, pp. 1885-1897.

[27] Okunlola, O. A., and Ocan, O. O. (2002). The expected environmental impact and mitigation studies of organized mining of rare metal $(\mathrm{Ta}-\mathrm{Sn}-\mathrm{Nb})$ pegmatites around Keffi area. North central Nigeria. J.Env. Ext. 3; 64-68

[28] Okunlola, O.A and Oyedokun, M.O. (2009). Compositional trends and rare metal (Ta-Nb) mineralization potential of pegmatite and association lithologies of Igbeti area, Southwestern Nigeria. Jour. RMZ- Materials And Geoenvironment, Vol.56, No.1, pp. 38-53.

[29] Okunlola, O.A. (2005). Metallogeny of Tantalite-Niobium Mineralization of Precambrian Pegmatites of Nigeria. Mineral Wealth; 104/2005, pp. 38-50.

[30] Okunlola, O.A. and Jimba, S. (2006). Compositional trends in relation to $\mathrm{Ta}-\mathrm{Nb}$ mineralization in Precambrian pegmatites of Aramoko and Ara-Ijero area, southwestern Nigeria. Journal of Mining and Geology; Vol. 42(2), pp. 113-126.

[31] Okunlola, O.A. and King, P.A. (2003). Process test work for the recovery of Tantalite-Columbite Concentrates from rare metal pegmatites of Nasarawa area, central Nigeria. Global Jour. Geological Sci, 1 (1) pp. 85-103.

[32] Okunlola, O.A. and Somorin, E.B. (2005). Compositional features of Precambrian pegmatites of Itakpe area, central Nigeria. Global Journal Of Geological Sciences Vol.4 (2), pp. 221-230.

[33] Pearce, J.A. Harris, N.B.W. and Tindle, A.G. (1984). Trace element discriminant diagrams for the tectonic interpretation of granitic rocks. Jour.Petrol. Vol.25, pp. 956-983

[34] Piper, D.Z. (1974). Rare earth elements in sedimentary cycle: A summary. Chem.Geol. Vol.14, pp. 285304.

[35] Rudenko S. A., Romanov V.A., Morakhovskyi V.N., Tarasov E. B., Galkin G. A., Dorokin V. K. (1975). Conditions of formation and controls of distribution of muscovite objects of the NorthBaikal muscovite province, and some general problems of pegmatite consolation. In: Gordiyenko V.V. (ed.) Muscovite pegmatites of the USSR, Nauka, Leningrad, pp 174-182 (in Russia)

[36] Staurov, O.D., Stolyarov, L.S. and Isochewa, E.I. (1969). Geochemistry and Origin of Verkh Iset Granitoid massif in central Ural. Geochem. Intern. Vol.6, pp. 1138-1146.

[37] Taylor, S.R., Rudnick, R.L., Mc Lennan, S.C and Eriksson, K.A. (1986). Rare earth element patterns in Archean high-grade metasediments and their tectonic significance. Geochim.Cosmochim. Acta, 50, pp. 2267-2279.

Asian Business Consortium realizes the meaning of fast publication to researchers, particularly to those working in competitive and dynamic fields. Hence, we offer an exceptionally fast publication schedule including prompt peer-review by the experts in the field and immediate publication upon acceptance. 


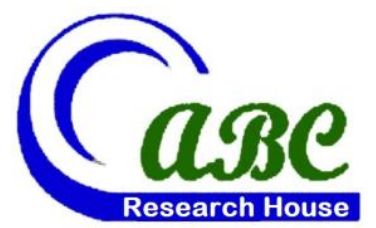

- Rd 4, Shyamoli, Dhaka-1207, Bangladesh

- Pantidalam, Kuala Lampur, Malaysia

- 3900 Woodhue Place, Alexandria, VA 22309, USA

www.abcreorg.weebly.com / www.abcjournals.net

Asian Business Consortium (ABC) is a multi-disciplinary research, training, publishing, digital library supporting and service house. Though founded in 2010 as the Business and Computing organization of Asia, it was reconstituted as the ABC in 2011. It has been working for creating and nurturing talents in USA, Malaysia and Bangladesh since its inception. As ABC is going global, it intends to open chapters in Australia, Germany, Japan, Pakistan, and other Asian countries in near future. The objectives of consortium are solely centered round the welfare and humane attitude of the founders who enthusiastically took up this noble cause and materialized it with a view to promote research and educational activities for the encouragement of scholars to develop their knowledge, to publish their analysis oriented scientific researches in international Journals, books, the task of organizing workshops, seminars, conferences, training, personality development programs and allied services.

In addition to research activities, ABC provides a good number of scholarships to the poor and meritorious students at various levels of education throughout the world. It plays an important role in the field of research by funding research projects and publishing the research papers. This consortium will unquestionably become the mouth-piece of the dark horses and unacknowledged scholar whose endowed and commendable contributions shall be provided an outlet keeping in mind the greater good of the larger society of the world.

$\mathrm{ABC}$ runs the following international referred journals for creating a platform to share the thoughts of professionals, scholars and academicians throughout the world.

\section{ABC Publications (ABC Journals)}

- Asian Accounting and Auditing Advancement (4A Journal); ICV 5.09

- $\quad$ Asian Business Review (ABR); UIF 0.1809

- Asian Journal of Applied Sciences and Engineering (AJASE); UIF 0.6351

- Global Disclosure of Economics and Business (GDEB)

- $\quad$ ABC Journal of Advanced Research (ABC-JAR)

- International Journal of Reciprocal Symmetry and Theoretical Physics (IJRSTP)

- American Journal of Trade and Policy (AJTP)

- Asian Journal of Humanity, Art and Literature (AJHAL)

- $\quad$ ABC Journal of Medical and Biological Research (ABC-JMBR)

- Asia Pacific Journal of Energy and Environment (APJEE)

- $\quad$ Engineering International (EI)

- $\quad$ ABC Research Alert (Online)

Each journal home page provides specific information for potential authors and subscribers. Open access policy, the quick review process, rich editorial boards and quality publications have already made $A B C$ Journals unique. ABC Journals are published under the direct supervisions of renowned academicians of the world.

Collaboration in Conference: $A B C$ considers high-quality conference papers for publication. Please contact us for detailed information.

Collaboration in Publishing: If you like to start writing a book, propose a new journal or advertise in $\mathrm{ABC}$ journals, please feel free to contact us. 


\section{Submit your next manuscript at- www.abcjournals.net}

ABC Journals is a unique forum to offer open access to all of its articles.

Now ABC Journals's portfolio is over ten journals, which publish both online and in print. 